\title{
Regularizing firing patterns of rat subthalamic neurons ameliorates parkinsonian motor deficits
}

\author{
Qian-Xing Zhuang, ${ }^{1}$ Guang-Ying Li, ${ }^{1}$ Bin Li, ${ }^{1}$ Chang-Zheng Zhang, ${ }^{1}$ Xiao-Yang Zhang, ${ }^{1}$ Kang Xi, ${ }^{1}$ Hong-Zhao Li, ${ }^{1}$ \\ Jian-Jun Wang, ${ }^{1,2}$ and Jing-Ning Zhu' ${ }^{1,2}$ \\ 'State Key Laboratory of Pharmaceutical Biotechnology and Department of Physiology, School of Life Sciences, and ${ }^{2}$ Institute for Brain Sciences, Nanjing University, Nanjing, China.
}

\begin{abstract}
The subthalamic nucleus (STN) is an effective therapeutic target for deep brain stimulation (DBS) for Parkinson's disease (PD), and histamine levels are elevated in the basal ganglia in PD patients. However, the effect of endogenous histaminergic modulation on STN neuronal activities and the neuronal mechanism underlying STN-DBS are unknown. Here, we report that STN neuronal firing patterns are more crucial than firing rates for motor control. Histamine excited STN neurons, but paradoxically ameliorated parkinsonian motor deficits, which we attributed to regularizing firing patterns of STN neurons via the hyperpolarization-activated cyclic nucleotide-gated channel 2 (HCN2) channel coupled to the H2 receptor. Intriguingly, DBS increased histamine release in the STN and regularized STN neuronal firing patterns under parkinsonian conditions. HCN2 contributed to the DBS-induced regularization of neuronal firing patterns, suppression of excessive $\beta$ oscillations, and alleviation of motor deficits in PD. The results reveal an indispensable role for regularizing STN neuronal firing patterns in amelioration of parkinsonian motor dysfunction and a functional compensation for histamine in parkinsonian basal ganglia circuitry. The findings provide insights into mechanisms of STN-DBS as well as potential therapeutic targets and STN-DBS strategies for PD.
\end{abstract}

\section{Introduction}

The subthalamic nucleus (STN), the only excitatory structure in the basal ganglia, holds a key position in the basal ganglia circuitry and is an optimal clinical target for deep brain stimulation (DBS) $(1,2)$ for treatment of motor symptoms in Parkinson's disease (PD). The STN not only constitutes the classic indirect pathway (3-5) and the hyperdirect cortico-STN-pallidal pathway (6-8), but also serves as a central pacemaker for the basal ganglia circuitry (9). Although the hypothesis of activating (cortical) afferents/passing fibers to the STN has been proposed and prevalently accepted $(10,11)$, the precise therapeutic mechanisms of STN-DBS remain undefined, which constrains the optimization and development of DBS strategies. Notably, in PD patients (12-16) and nonhuman primate $(3,17,18)$ and rodent $(11,19-21)$ models of $\mathrm{PD}$, abnormal irregular neuronal firing rates, firing patterns, and network oscillations are recorded in the STN, indicating a close relationship between the abnormal STN neuronal activities and parkinsonian motor deficits. Yet little is known about the intrinsic properties and endogenous modulators responsible for modulation of STN neuronal activities. Uncovering these intrinsic and endogenous substrates will help with understanding the pathophysiology of PD and the neuronal mechanism underlying STN-DBS.

\section{Related Commentary: p. 5201}

Authorship note: QXZ, GYL, and BL contributed equally to this work. Conflict of interest: The authors have declared that no conflict of interest exists. License: Copyright 2018, American Society for Clinical Investigation. Submitted: January 23, 2018; Accepted: September 11, 2018. Reference information: / Clin Invest. 2018;128(12):5413-5427. https://doi.org/10.1172/JCI99986.
Intriguingly, a significant increase in histamine levels and a functional alternation in the histaminergic system in the basal ganglia are revealed in PD patients (22-24). Central histamine, restrictedly synthesized in the tuberomammillary nucleus (TMN) of the hypothalamus, has been well known to be a general modulator for whole-brain functions $(25,26)$, including not only sleep and wakefulness $(27,28)$, but also motor control $(29,30)$. Nevertheless, the role of histamine and the central histaminergic system in basal ganglia motor function and PD pathophysiology is controversial. Since its level is elevated in PD, histamine has been implicated in either deterioration of PD or sleep disorders and nonmotor dysfunction in PD, but is less associated with motor symptoms $(31,32)$. Here, we report, however, that the endogenous histamine/histaminergic system is essential for regularizing STN neuronal firing patterns, which are more crucial than firing rates for maintenance of normal basal ganglia motor function. The hyperpolarization-activated cyclic nucleotide-gated (HCN) channel 2 contributes to regularization of firing patterns of STN neurons by, not only histaminergic inputs, but also high-frequency DBS, both of which ameliorate parkinsonian motor deficits. Regularization of firing patterns of STN neurons in PD may account for why STN-DBS restores motor function in PD.

\section{Results}

Histamine levels are gradually increased in STN of $P D$ rats, and histamine ameliorates PD motor impairment. We first detected histamine levels in bilateral STNs (Figure 1A) in the 6-hydroxydopamine-lesioned (6-OHDA-lesioned) rat model of hemiparkinsonian (Supplemental Figure 1A; supplemental material available online with this article; https://doi.org/10.1172/ JCI99986DS1). The histamine level in the ipsilesional STN (Fig- 
A

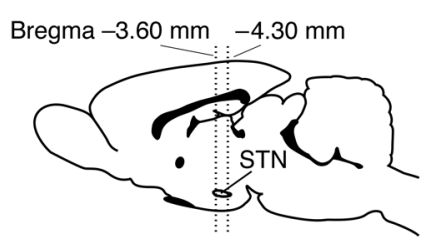

B

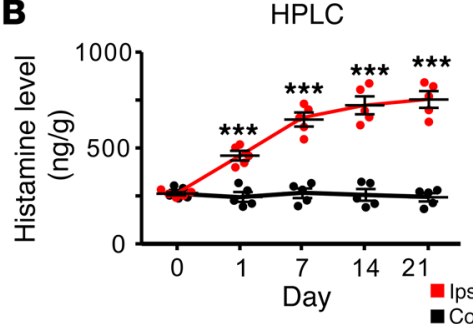

ELISA

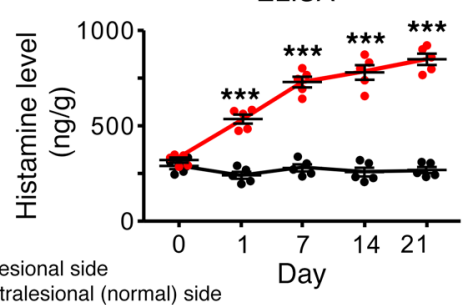

C
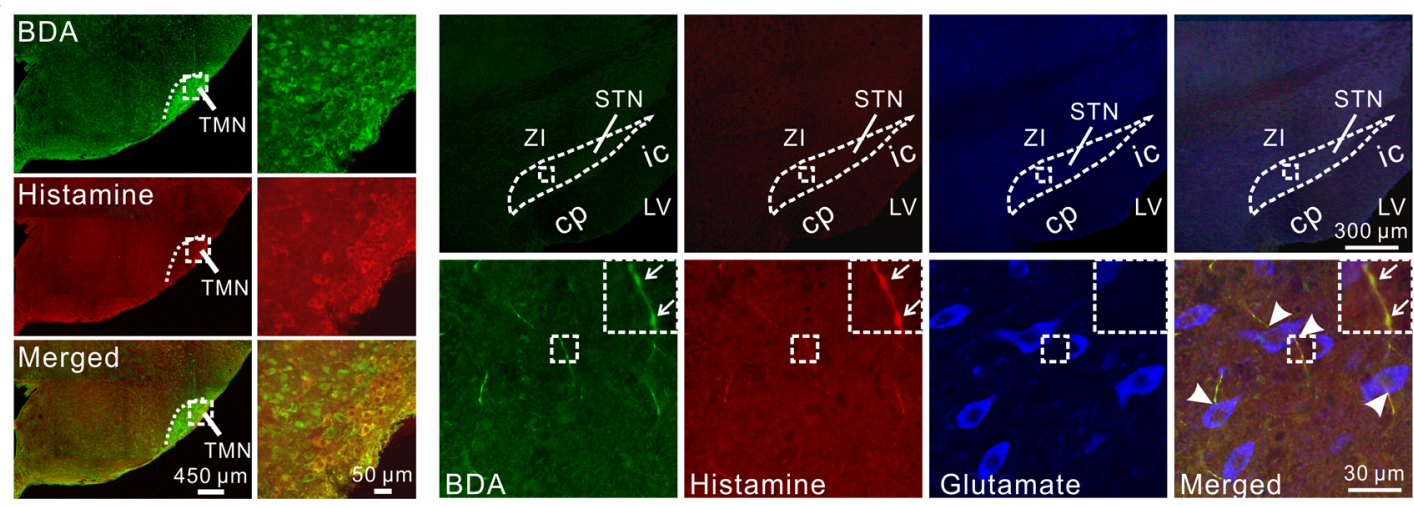

D
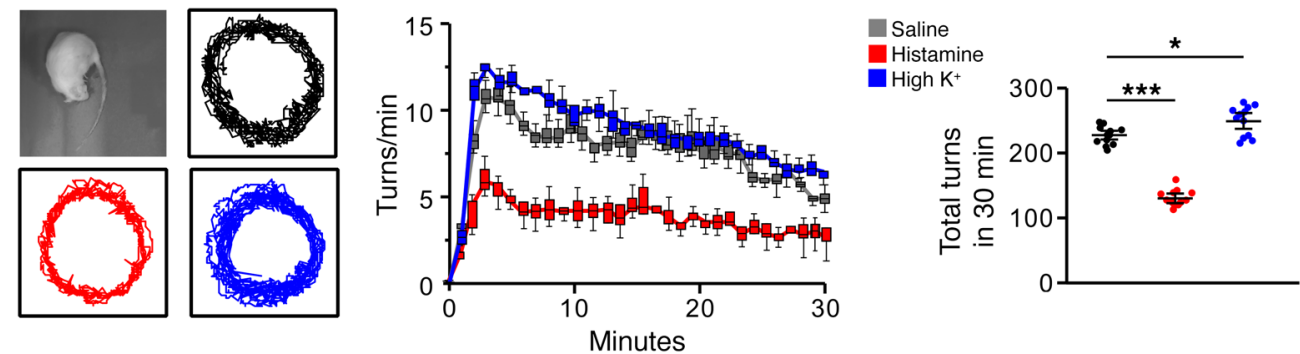

Figure 1. Histaminergic afferents in the STN and the histamine-induced amelioration of turning behavior of PD rats. (A) Sagittal view of the rat brain showing the localization of the STN between -3.60 and $-4.30 \mathrm{~mm}$ from the bregma. STN tissue punches for analysis of histamine levels were collected from brain slices obtained using these coordinates. (B) HPLC and ELISA analyses show levels of histamine (ng/g of tissue) in the ipsilesional and contralesional STN of PD rats $(n=10)$ on $1,7,14$, and 21 days after 6 -OHDA injection $(n=5)$. (C) Immunofluorescence staining shows that anterogradely labeled BDA fibers in the STN, originating from the histaminergic neurons in the hypothalamic TMN (left panels), contained histamine immunoreactivity (right panels). Note that these histaminergic fibers possessed prominent varicosities (indicated by arrows) and passed around (indicated by arrowheads) glutamate immunoreactive (glutamatergic) neurons in the STN (3 independent experiments). cp, cerebral peduncle; ic, internal capsule; LV, lateral ventricle; ZI, zona incerta. (D) Behavioral tests show that histamine $(1 \mu \mathrm{g})$ microinjected into STN decreased, whereas high $\mathrm{K}^{+}(0.75 \mu \mathrm{g} \mathrm{KCl})$ increased, the rate and total number of apomorphine-induced turnings in 30 minutes in PD rats $(n=12)$. Data are represented as mean \pm SEM or median (horizontal bar) with 25 th-75th (box) and 5th-95th (whiskers) percentiles. ${ }^{*} P<0.05$; ${ }^{* *} P<0.001$, 2-way (B) or 1-way ANOVA (D) with Newman-Keuls post hoc test.

ure 1B) was gradually increased with the damage of dopaminergic neurons (Supplemental Figure 1B) and the decrease of dopamine levels (Supplemental Figure 1C) in the substantia nigra, whereas that in the contralesional STN remained normal. Mapping the histaminergic projections in the STN by anterograde tracings showed that the histamine-immunoreactive fibers originating from the histaminergic neurons in the TMN were scattered throughout the STN (Figure 1C). These histaminergic fibers possessed prominent varicosities and passed around glutamatergic neurons in the STN (Figure 1C), suggesting that a neural modulation on STN neurons may occur through histamine released from the histaminergic terminals. Concurrent with the increase in histamine level, the histaminergic fiber density was also increased in the ipsilesional rather than contralesional STN (Supplemental Figure 1D) in PD rats.
Histamine is known as a homogeneous excitatory modulator on various brain regions $(25,26)$. According to the classic model of basal ganglia $(5,33)$, increase in STN neuronal firing rates leads to enhancing the activity of indirect pathway to inhibit movement. Thus, if histamine excites STN neurons, the seemingly logical conclusion is that the excitatory modulation of histamine on STN results in deteriorating motor deficits in PD. However, surprisingly, unlike high $\mathrm{K}^{+}$, histamine locally microinjected into the ipsilesional STN decreased apomorphine-induced turnings in PD rats (Figure 1D), i.e., ameliorated the parkinsonian motor impairment.

Histamine rather than high $\mathrm{K}^{+}$regularizes firing patterns of STN neurons in PD rats both in vivo and in vitro. We were curious about the mechanism underlying the amelioration effect of histamine on parkinsonian motor dysfunction. We examined the effect of histamine on single-unit firing in STN by spike sorting and analysis 

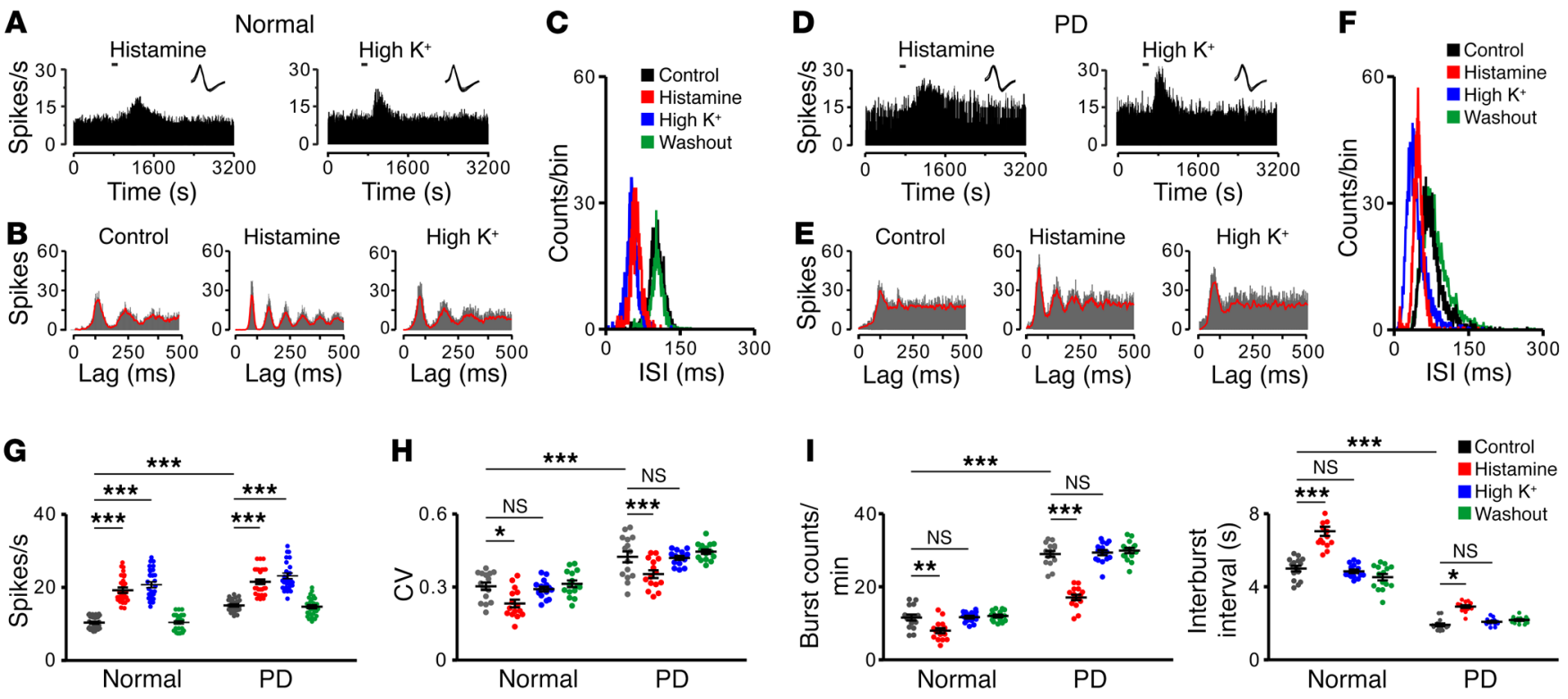

Figure 2. The histamine-induced regularization of firing patterns of STN neurons in normal and PD rats. (A-F) Effects of histamine $(1 \mu \mathrm{g})$ and high $\mathrm{K}^{+}$ $(0.75 \mu \mathrm{g} \mathrm{KCl})$ on firing rate and firing pattern of 2 recorded STN neurons in normal and PD rats in vivo. PSTHs (A and $\mathbf{D})$ show that both histamine and high $\mathrm{K}^{+}$excited the STN neuron. Insets represent 5 superimposed traces of spike waveforms for each unit, respectively. Autocorrelation histograms (B and E) show that histamine, rather than high $\mathrm{K}^{+}$, promoted periodicity of STN neuronal firing. ISI histograms (C and $\mathbf{F}$ ) show that histamine, but not high $\mathrm{K}^{+}$, narrowed ISI distributions. (G-I) Histamine increased firing rates $(\mathbf{G}, n=30)$, decreased CV of ISIs $(\mathbf{H}, n=15)$, reduced number of bursts (I, left panel, $n=15)$, and prolonged the interburst intervals (I, right panel, $n=15)$ of STN neurons in both normal and PD rats. However, high $\mathrm{K}^{+}$only increased firing rates $(\mathbf{G})$, but did not influence firing patterns ( $\mathbf{H}$ and $\mathbf{I})$ of STN neurons in normal and $\mathrm{PD}$ rats. Data are represented as mean $\pm \mathrm{SEM}$. ${ }^{*} P<0.05 ;{ }^{* *} P<0.01$; ${ }^{* * *} P<0.001,2$-way ANOVA with Newman-Keuls post hoc test (G-I).

of multichannel recordings in vivo. As expected, both histamine and high $\mathrm{K}^{+}$induced a significant increase in firing rates of STN neurons in normal and PD rats (Figure 2, A, D, and G). But intriguingly, by analyzing unit firing autocorrelograms (Figure 2B), interspike interval (ISI) histograms (Figure 2C), and coefficient of variation (CV) of ISIs (Figure 2H), we found that histamine, instead of high $\mathrm{K}^{+}$, increased periodicity of STN neuronal firing, narrowed ISI distributions, and decreased the CV of ISIs in normal rats. These results suggest that histamine may regularize firing patterns of STN neurons. Compared with those in normal rats, STN neurons in PD rats exhibited an increase in firing rates (Figure 2G) and a concomitantly irregular firing pattern, with a loss of periodicity of discharges (Figure 2, B and E), altered ISI distributions (Figure 2, C and F), and increased CV of ISIs (Figure 2H) as well as an increased number of bursts and shortened interburst intervals (Figure 2I), which are in accord with previous observations in both PD patients and animal models (3, 34-36). Notably, histamine significantly restored STN neuronal firing patterns in parkinsonian conditions both in vivo (Figure 2, E, F, H, and I) and in vitro (Supplemental Figure 2), but high $\mathrm{K}^{+}$had no such effect. Therefore, we suggest that regularization of firing patterns of STN neurons may account for why histamine ameliorates motor dysfunction in PD.

$H 2$ receptor and its coupled HCN channel mediate the histamine-induced regularization of STN neuronal firing patterns and amelioration of $P D$ motor deficits. We assessed receptor and ionic mechanisms underlying the effect of histamine on STN neurons by whole-cell patch clamp recordings in vitro (Figure 3A). Histamine directly induced an inward current on STN neurons in a concentration-dependent manner (Supplemental Figure 3, A and B) and did not influence miniature excitatory postsynaptic currents (EPSCs) and inhibitory postsynaptic currents (IPSCs) (Supplemental Figure 3, C and D), suggesting a direct postsynaptic effect of histamine. This effect was mediated by postsynaptic histamine $\mathrm{H} 2$ rather than $\mathrm{H} 1$ and $\mathrm{H} 4$ receptors (Supplemental Figure 3, $\mathrm{E}$ and F). Single-cell quantitative PCR (qPCR) and immunofluorescence results confirmed that only the $\mathrm{H} 2$ receptor was expressed and localized in STN neurons (Supplemental Figure 4). Furthermore, the membrane conductance of STN neurons exhibited a significant feature of hyperpolarization activation (Figure 3B), and the hyperpolarization-activated current was reversed at $-31 \mathrm{mV}$ (Supplemental Figure $5 \mathrm{~A}$ ), which is near the reversal potential of the HCN channel (37). Histamine increased, not only the hyperpolarization-activated conductance (Figure 3B), but also the depolarizing sag, which is triggered by activation of the HCN channel. ZD7288 $(50 \mu \mathrm{M})$, a selective blocker for the HCN channel, abolished the depolarizing sag (Figure 3C) and the histamine-induced hyperpolarization-activated inward current (Supplemental Figure $5 \mathrm{~B})$, which was also blocked by ranitidine, a selective antagonist for the $\mathrm{H} 2$ receptor (Figure 3D). Thus, the HCN channel coupled to the $\mathrm{H} 2$ receptor mediates the effect of histamine on STN neurons. More importantly, both ranitidine and a low concentration of ZD7288 $(1 \mu \mathrm{M})$ effectively blocked the histamine-induced increase in the regularity of STN neuronal firing patterns in normal rats (Figure 3, E-G), indicating that the $\mathrm{HCN}$ channel is responsible for regularizing firing patterns of STN neurons by histamine.

To further determine whether the $\mathrm{H} 2$ receptor and its coupled HCN channel mediate the histamine-induced amelioration of motor deficits in $\mathrm{PD}$, we evaluated motor performances by 
A

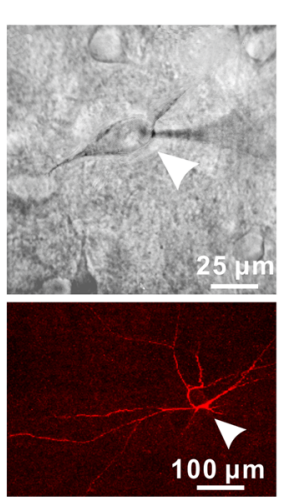

B

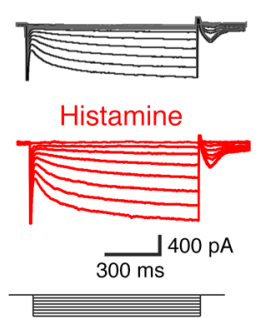

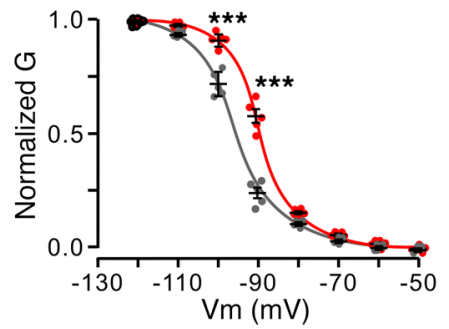

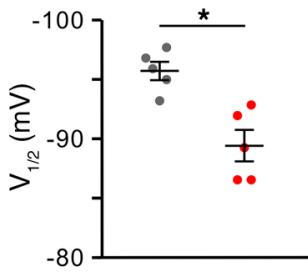

C
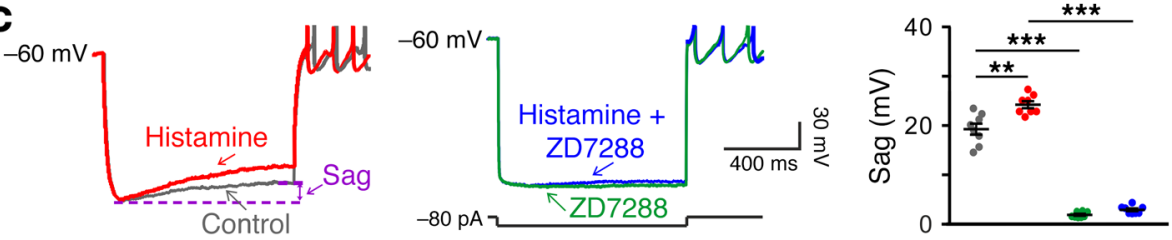

D
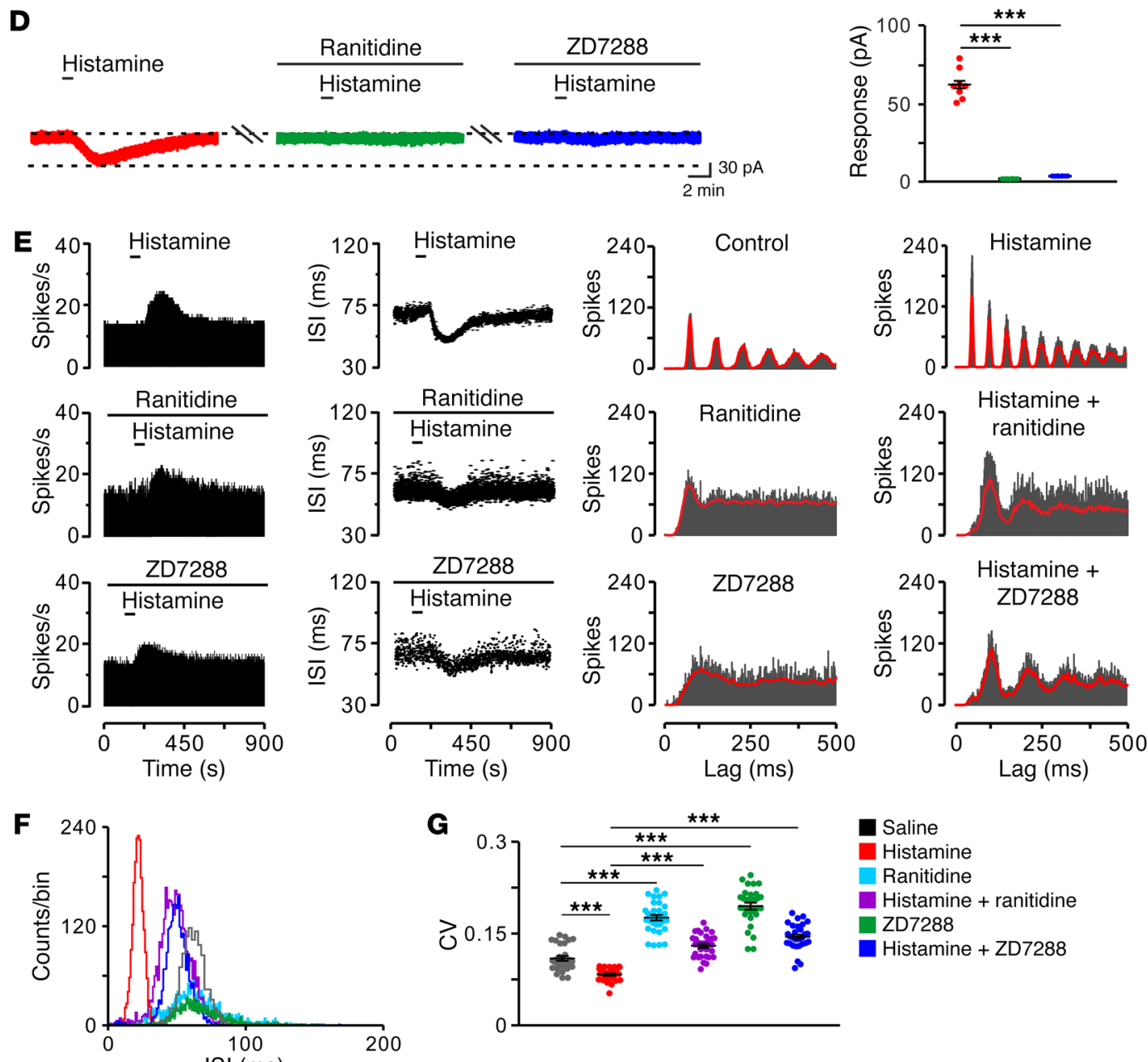

ISI (ms)

Figure 3. HCN channel coupled to H2 receptor mediates the effect of histamine on STN neurons in normal rats. (A) Microscope image of a STN neuron (indicated by arrowheads) recorded in a brain slice and labeled with biocytin after patch-clamp recording. (B) Histamine (10 $\mu \mathrm{M})$ shifted the conductance-voltage curve of recorded STN neurons (at $-90 \mathrm{mV}$ and $-100 \mathrm{mV}, n=5$ ). The conductance curve was converted from the whole-cell currents recorded from -50 to $-120 \mathrm{mV}$ and was fitted by Boltzmann function. Note that the conductance exhibited a significant feature of hyperpolarization activation and histamine reduced the voltage required for half-maximal activation $\left(V_{1 / 2}, n=5\right)$. (C) Histamine increased the inward rectification (sag) in response to an 80 pA hyperpolarizing current pulse. ZD7288 $(50 \mu \mathrm{M})$, a selective blocker for the HCN channel, abolished the depolarizing sag in both the absence and presence of histamine $(n=8)$. (D) Histamine elicited an inward current in a STN neuron, and ranitidine $(1 \mu M)$, a selective antagonist for the H2 receptor, or ZD7288 $(50 \mu \mathrm{M})$ totally blocked the current induced by histamine $(n=8)$. (E and F) PSTHs, scatter plots of ISI series, autocorrelation histograms, and an ISI histogram of discharges of a recorded STN neuron show the histamine-induced changes in firing rate and firing pattern in the absence and presence of ranitidine and ZD7288 $(1 \mu \mathrm{M}$, respectively). (C) Group data show that histamine significantly decreased the CV of ISIs, whereas ZD7288 remarkably increased the CV and blocked the histamine-induced decrease in CV $(n=30)$. Data are represented as mean $\pm \mathrm{SEM} .{ }^{*} P<0.05 ;{ }^{* *} P<0.01 ;{ }^{* *} P<0.001$, 2 -tailed paired $t$ test (B) or 1-way ANOVA with Newman-Keuls post hoc test (C, D, and $\mathbf{G}$ ). 
means of pharmacological manipulation of histaminergic modulation of STN. Electrophysiological and histological methods were employed to identify microinjection sites and effective spread in the STN (Supplemental Figure 6). Microinjected histamine significantly increased the firing rate and decreased the CV of ISIs on STN neurons close to the injection sites, whereas ranitidine decreased the firing rate and increased the CV of ISIs by antagonizing the H2 receptor to block endogenous histaminergic inputs (Supplemental Figure 6B). Neither histamine nor ranitidine influenced neurons in the border between STN and zona incerta (Supplemental Figure 6, A and C), indicating a manipulation confined within the STN. As shown in Figure 4, blocking histaminergic inputs by ranitidine in STN significantly increased the apomorphine-induced turnings in PD rats, whereas dimaprit, a selective agonist for the $\mathrm{H} 2$ receptor, mimicked the histamine-induced decrease in turnings (Figure 4A). However, microinjection of antagonists or agonists for the H1, $\mathrm{H} 3$, or $\mathrm{H} 4$ receptor into STN had no effect on the turning behavior of PD rats (Supplemental Figure 7), which is consistent with our above-mentioned electrophysiological and single-cell qPCR results. Blocking the HCN channel by ZD7288 not only increased the turnings of PD rats, but also abolished the histamine-induced decrease in turnings (Figure 4A). In addition, we assessed slowness of motor initiation and execution of PD rats by the adhesiveremoval test (Figure 4B) and gait disturbance by the footprint test (Figure 4C). We found that microinjection of histamine into STN significantly shortened the prolonged time for removing a rectangular adhesive strip from the contralesional forelimb (Figure 4B) and enlarged bilateral stride lengths instead of stride width (Figure $4 \mathrm{C})$. On the other hand, the effects of histamine were mimicked by dimaprit and attenuated by ranitidine and ZD7288 (Figure 4, $\mathrm{B}$ and $\mathrm{C})$. These results suggest that histaminergic modulation of STN may remedy bradykinesia and gait disturbance in PD via the $\mathrm{H} 2$ receptor and $\mathrm{HCN}$ channel. Therefore, the $\mathrm{H} 2$ receptor and its coupled HCN channel contribute to the histamine-induced amelioration of parkinsonian motor deficits.

Given the fact that histamine regularizes firing patterns of STN neurons and restores PD motor deficits, we speculate that a gradual rise of histamine levels in STN in PD progression may be a compensatory result for abnormal irregular firing patterns of STN neurons and that the enhanced histaminergic modulation may help to improve normal motor function of the basal ganglia. As expected, in normal rats, bilateral microinjection of histamine into STN promoted motor performances in the accelerating rotarod and balance beam and blockage of endogenous histaminergic inputs by blockage of the $\mathrm{H} 2$ receptor or $\mathrm{HCN}$ channel attenuated motor performances (Supplemental Figure 8). Therefore, histaminergic modulation may serve as an indispensable biasing force for the basal ganglia circuity, which not only maintains normal motor functions, but also alleviates parkinsonian motor dysfunctions.

The HCN2 channel is responsible for the amelioration of motor deficits in PD rats by histamine. In mammals, $4 \mathrm{HCN}$ channel subtypes have been identified (38-40), and all of them were expressed and localized in the STN (Supplemental Figure 9). Interestingly, the activation curve for HCN conductance in STN neurons showed that the channel was activated slowly at hyperpolarized potentials more negative than $-70 \mathrm{mV}$ (Figure 3B) and that the membrane potential for half-maximal activation $\left(V_{1 / 2}=95.8 \pm\right.$
$0.86 \mathrm{mV})$ was closer to that of the cloned HCN2 $(40,41)$ and was significantly shifted by histamine toward the depolarizing direction (Figure 3B). The results indicate an activation of the HCN2 channel by histamine on STN neurons. To further assess the contribution of each HCN channel subtype to the histamine-induced motor improvements in PD rats, we generated lentiviral vectors to separately downregulate the expression of each subtype of the HCN channel in STN (Figure 5, A-D, Supplemental Figure 10, and Supplemental Table 1). Properties of the HCN channel in STN neurons did not change in PD (Supplemental Figure 11, A, B, D, and $\mathrm{E}$ ), but HCN2 downregulation decreased the depolarizing sag and HCN current in STN neurons (Supplemental Figure 11, C-E). Accordingly, downregulation of HCN2 significantly increased apomorphine-induced turnings (Figure 5F), prolonged contralesional rather than ipsilesional adhesive-removal time (Figure 5G and Supplemental Figure 12A), and shortened stride length instead of stride width (Figure 5H and Supplemental Figure 12B) in PD rats, whereas downregulation of $\mathrm{HCN} 1, \mathrm{HCN} 3$, or HCN4 had no effect on these motor deficits (Figure 5, F-H). Moreover, the histamine-induced motor improvements in $\mathrm{PD}$ rats were blocked by downregulation of $\mathrm{HCN} 2$ rather than $\mathrm{HCN} 1, \mathrm{HCN} 3$, or HCN4 (Figure 5, F-H). On the other hand, overexpression of the HCN2 channel in STN (Figure 5E) not only restored motor deficits of PD rats, but also promoted histamine-induced improvements (Figure 5, F-H, and Supplemental Figure 12). In normal rats, downregulation of $\mathrm{HCN} 2$ rather than $\mathrm{HCN} 1, \mathrm{HCN} 3$, or HCN4 remarkably attenuated motor performances on an accelerating rotarod and a balance beam and also attenuated histamine-induced motor improvements (Supplemental Figure 13, A and B), whereas overexpression of HCN2 significantly improved motor performances and increased histamine-induced improvements (Supplemental Figure 13, C and D). Since the HCN2 channel is colocalized with the $\mathrm{H} 2$ receptor on STN neurons (Supplemental Figure 14) and selectively expressed in the glutamatergic instead of GABAergic neurons in the STN (Supplemental Figure 15), we suggest that HCN2 rather than the HCN1, -3 , and - 4 channels may be coupled to the $\mathrm{H} 2$ receptor and contribute to the histaminergic modulation of STN glutamatergic projection neurons and the consequent amelioration of parkinsonian motor dysfunction and improvement of normal motor function.

The HCN2 channel contributes to the DBS-induced regularization of STN neuronal firing patterns, suppression of $\beta$ oscillations, and alleviation of motor deficits in PD rats. We further determined the role of the HCN2 channel in the regularization of firing patterns of STN neurons under parkinsonian conditions. As shown in Figure 6, A-F, firing patterns of STN neurons in PD rats with overexpression of HCN2 in STN were more regular than those in untreated PD rats. In contrast, downregulation of $\mathrm{HCN} 2$ made STN neuronal firing patterns more irregular (Figure 6, A-F). It is noteworthy that overexpression of HCN2 in STN neurons ameliorated motor deficits, whereas downregulation of HCN2 deteriorated motor dysfunctions (Figure 5, F-H). These results strongly indicate that the HCN2 channel may play an important role in regularizing firing patterns of STN neurons, which is critical for basal ganglia motor functions. Considering that STN is an optimal target for clinical DBS treatment for PD and that abnormal irregular firing patterns are recorded in the STN neurons in PD patients $(34,42$, 

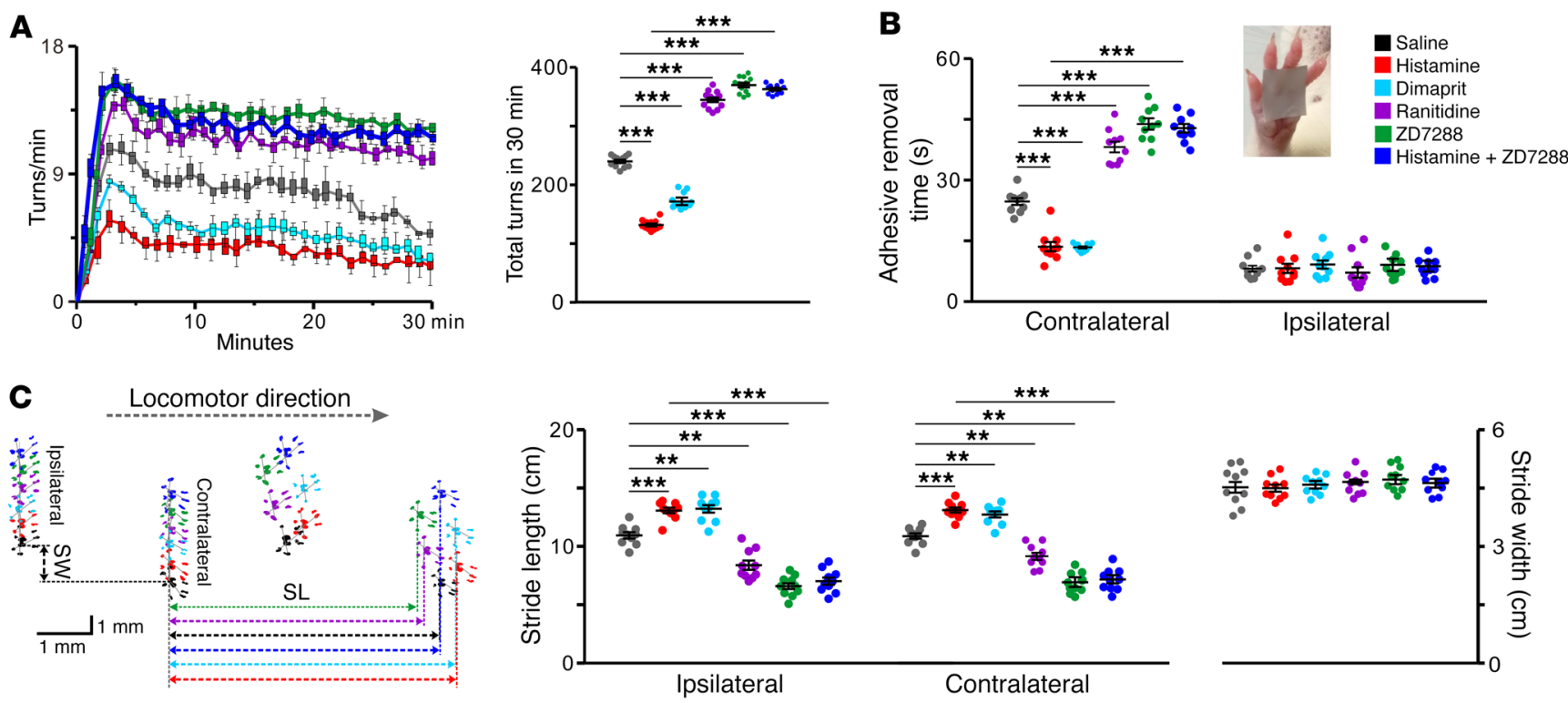

Figure 4. HCN channel coupled to $\mathrm{H} 2$ receptor mediates the histamine-induced amelioration of motor deficits in PD rats. Effects of microinjection of histamine $(1 \mu \mathrm{g}$ ), dimaprit (a selective agonist for $\mathrm{H} 2$ receptor, $2 \mu \mathrm{g}$ ), ranitidine (a selective antagonist for $\mathrm{H} 2$ receptor, $3.5 \mu \mathrm{g}$ ), and ZD7288 (a selective blocker for HCN channel, $3 \mu \mathrm{g})$ into the STN on turning behavior $(\mathbf{A}, n=12)$, adhesive-removal test $(\mathbf{B}, n=10)$, and locomotor footprints (C, $n=10)$ in PD rats. Dimaprit mimicked the histamine-induced decrease in turnings, shortened time for removing an adhesive strip from forelimb contralateral to the lesion, and enlarged bilateral stride lengths, whereas ranitidine and ZD7288 significantly increased the turnings, prolonged contralesional adhesive-removal time, and shortened bilateral stride lengths. ZD7288 also abolished the histamine-induced amelioration of turning, adhesive removal, and locomotor behaviors in PD rats. Data are represented as median (horizontal bar) with 25th-75th (box) and 5th-95th (whiskers) percentiles or mean \pm SEM. ${ }^{* *} P<0.01$; ${ }^{* * *} P<0.001$, 1-way (A and C, stride width) or 2-way ANOVA (B and C, stride length) with Newman-Keuls post hoc test.

43), we wondered whether DBS influences the regularity of STN neuronal firing patterns. Intriguingly, we found that high-frequency stimulation of STN remarkably regularized neuronal firing patterns of PD rats and that firing patterns became irregular again when DBS ceased (Figure 6, G-L), suggesting that regularization of firing patterns of STN neurons may account for the therapeutic effect of DBS on motor dysfunctions in PD.

Furthermore, we investigated the role of the HCN2 channel in STN-DBS-induced amelioration of motor dysfunction and regularization of STN neuronal firing patterns in free-moving PD rats. As shown in Figure 7A, high-frequency DBS of STN significantly increased the total movement distance of PD rats in an open field, as previously reported (11), indicating that STN-DBS effectively ameliorates parkinsonian locomotor impairment. In contrast, PD rats with downregulation of HCN2 did not exhibit improvements of locomotion during STN-DBS (Figure 7A), suggesting the HCN2 channel is critical for the DBS-induced remedy of locomotor deficit. Notably, the STN-DBS-induced motor improvement in free-moving PD rats was accompanied by an increase in histamine release in the STN (Figure 7B) and a regularization of firing patterns of numerous individual STN neurons (Figure 7, C-H) as well as a suppression of exaggerated oscillatory network synchronization at the $\beta$ band (15-25 Hz) (Figure 7, I and J), which is prevalent in the STN of animal models and patients with PD (44-46). Furthermore, downregulation of HCN2 abolished the STN-DBSinduced regularization of neuronal firing patterns in free-moving $\mathrm{PD}$ rats (Figure 7, C-H) and alleviation of excessive $\beta$ oscillations (Figure 7, I and J). All these results strongly suggest that the HCN2 channel may contribute to STN-DBS-induced regularization of
STN neuronal firing patterns and the consequent amelioration of motor deficits in PD.

\section{Discussion}

Both firing rates and firing patterns of STN neurons are implicated in the pathophysiology of PD $(3,17,21,36,47,48)$. Here, we demonstrate that firing patterns of STN neurons are more critical for basal ganglia motor function than firing rates. Increasing the firing rates of STN neurons, for instance, by administration of high $\mathrm{K}^{+}$, increases motor deficits of a 6-OHDA-lesioned rat model of PD (Figure 1D), which is in agreement with the classic direct/ indirect pathway model of basal ganglia $(3,5)$ and the alleviation of parkinsonian motor dysfunction by lesioning STN (17) or reducing STN neuronal activities $(49,50)$. On the contrary, histamine, which also exerts an excitatory effect on STN neuronal activity (Figure 2, A, D, and G), paradoxically ameliorates the apomorphine-induced turning, bradykinesia, and gait disturbance of $\mathrm{PD}$ rats (Figure 4). Considering that high $\mathrm{K}^{+}$does not influence firing patterns of STN neurons, but histamine significantly regularizes them (Figure 2), we propose the improvement of abnormal irregular STN neuronal firing patterns as a fundamental contributor to histamine-induced amelioration of parkinsonian motor deficits. Therefore, we suggest that the histaminergic modulation is crucial for maintenance of normal STN neuronal firing patterns and basal ganglia motor functions. The increase in histamine levels and histaminergic afferent density in PD progression may play a compensatory role for amelioration of abnormal STN neuronal firing patterns and parkinsonian motor deficits. In fact, several histaminergic agents have been empirically used in clinic for treatment, 
A LV-Hon1-shRNA
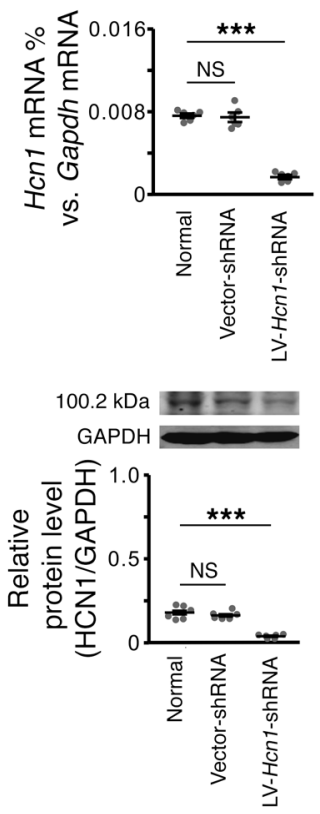

B LV-Hcn2-shRNA

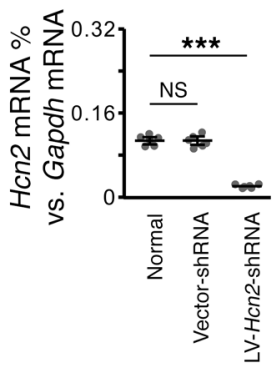

$94.7 \mathrm{kDa} \rightarrow \mathrm{mat}$ GAPDH

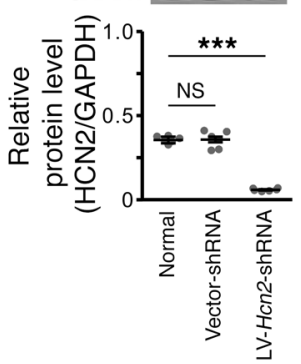

C LV-Hen3-shRNA
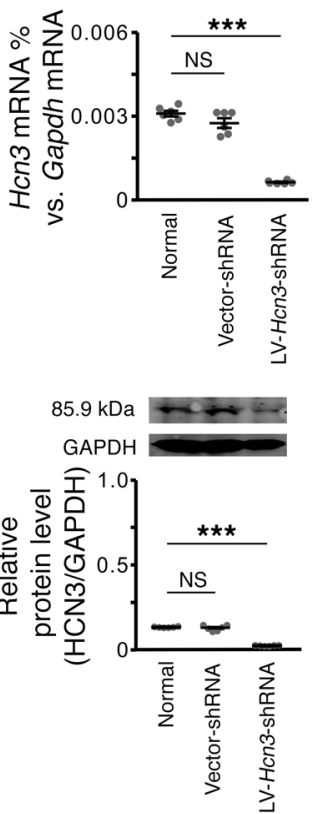

D LV-Hcn4-shRNA
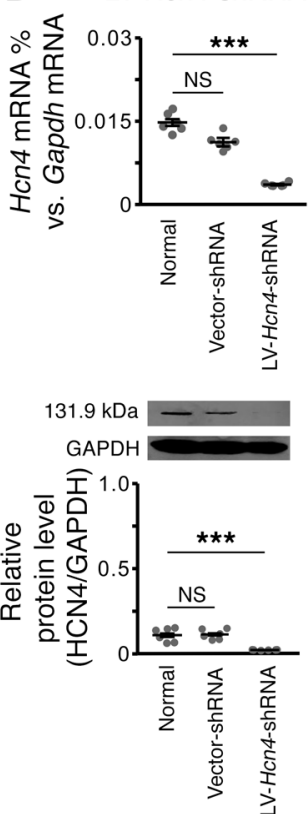

E LV-Hcn2-oe

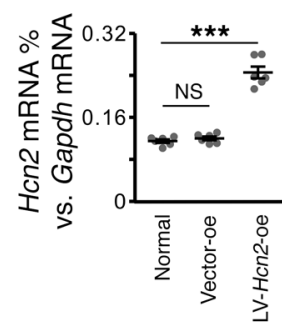

$94.7 \mathrm{kDa}+\cdots$

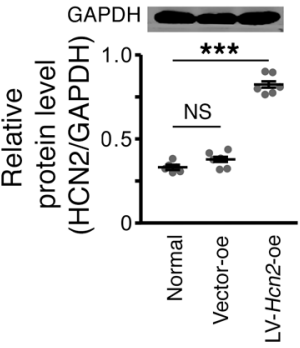

$\mathbf{F}$

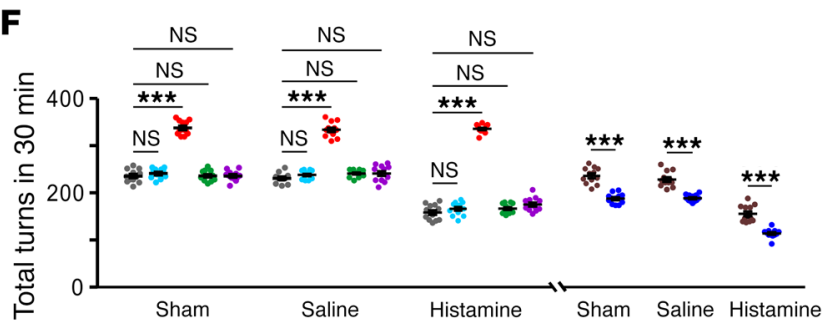

G

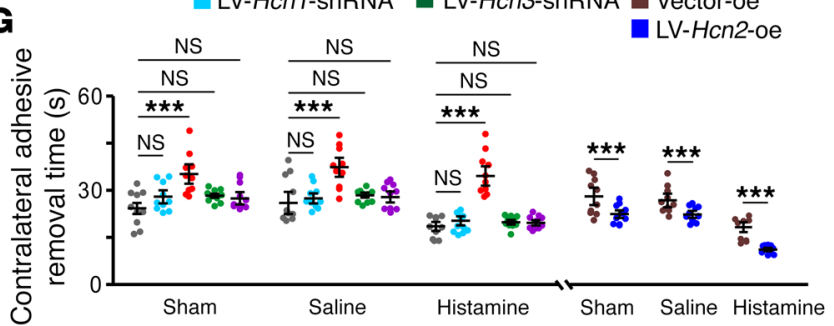

H

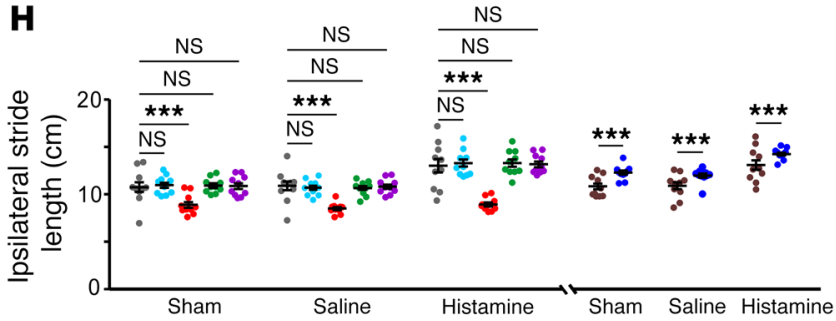

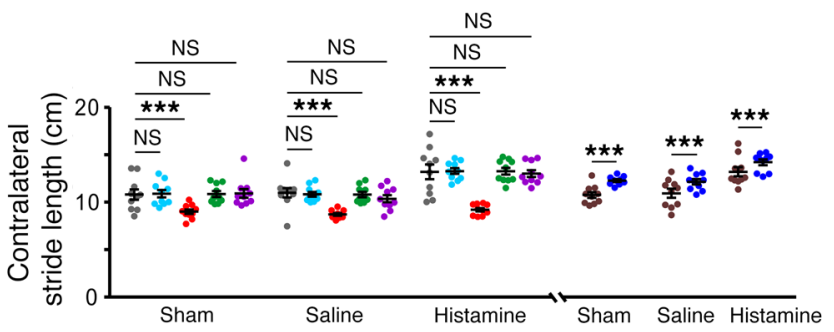

Figure 5. The HCN2 channel is responsible for the histamine-induced amelioration of motor deficits in PD rats. (A-D) LV-Hcn1-shRNA, LV-Hcn2-shRNA, LV-Hcn3-shRNA, and LV-Hcn4-shRNA effectively downregulated the expression of Hcn1, Hcn2, Hcn3, and Hcn4 mRNAs and proteins ( $n=6$ from 6 independent experiments) in the STN. (E) LV-Hcn2-oe upregulated the expression of Hcn2 mRNAs and proteins ( $n=6$ from 6 independent experiments). (F-H) Effects of downregulation and overexpression of the HCN2 channel in the STN on motor deficits of turning behavior (F, $n=12)$, adhesive-removal test (G, $n=10$ ), and locomotor footprints $(\mathbf{H}, n=10)$ in PD rats with sham operation, saline injection, and histamine injection. Downregulation of the HCN2 channel significantly increased the apomorphine-induced turnings, prolonged contralesional adhesive-removal time, and shortened bilateral stride length, whereas downregulation of the HCN1, HCN3, or HCN4 channel had no effect on these motor deficits. Only the downregulation of HCN2 rather than the HCN1, HCN3, or HCN4 channel blocked the amelioration of turnings, removal time, and stride length of PD rats induced by microinjection of histamine into the STN. Overexpression of the HCN2 channel in STN not only decreased the turnings, reduced removal time, and enlarged bilateral stride length of PD rats, but also improved the histamine-induced amelioration in these motor behaviors. Data are represented as mean $\pm \mathrm{SEM}$. ${ }^{* *} P<0.001,1$-way $(\mathbf{A}$-E) or 2 -way ANOVA (F-H) with Newman-Keuls post hoc test.

not only of parkinsonian nonmotor symptoms, but also of motor dysfunctions as adjuvant drugs $(51,52)$. Thus, restoration of normal firing patterns of STN neurons by targeting the histaminergic system and related ion channels may be an effective strategy for treatment of PD motor symptoms.
The HCN channel is well known as holding a key position in accelerating neuronal depolarization to generate pacemaking rhythmic activity and control firing patterns $(19,37)$. However, previous studies indicated that the HCN channel played minimal roles in the autonomous single-spiking firing of STN neurons (53, 


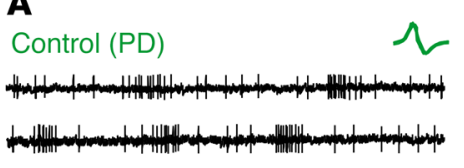

LV-Hcn2-oe (PD)
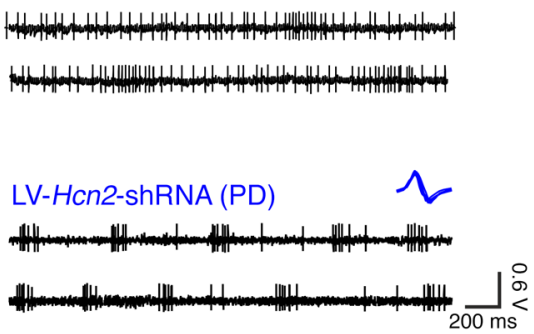

G

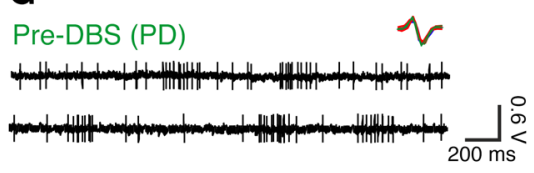

DBS (PD)

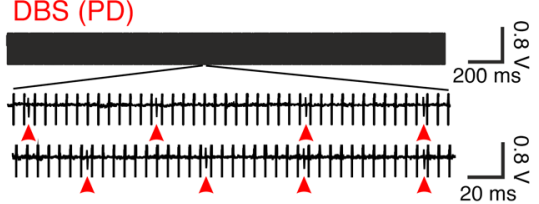

Post-DBS (PD)

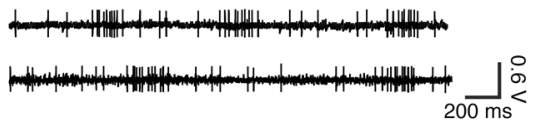

B
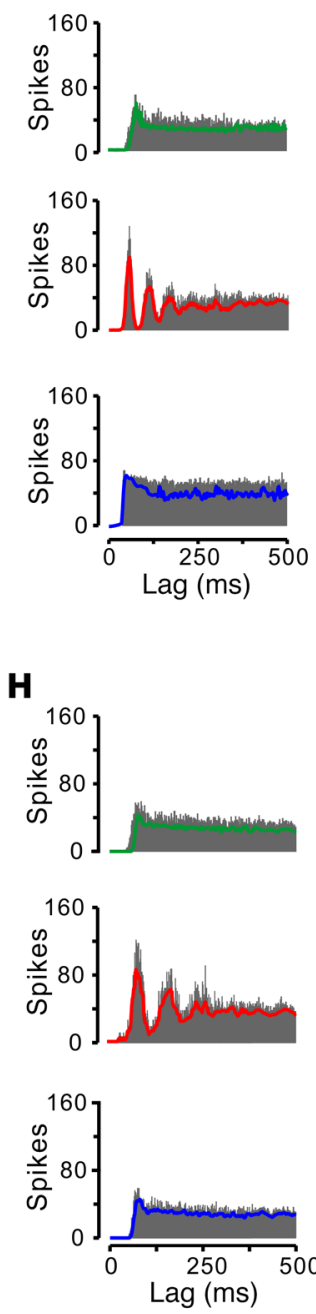
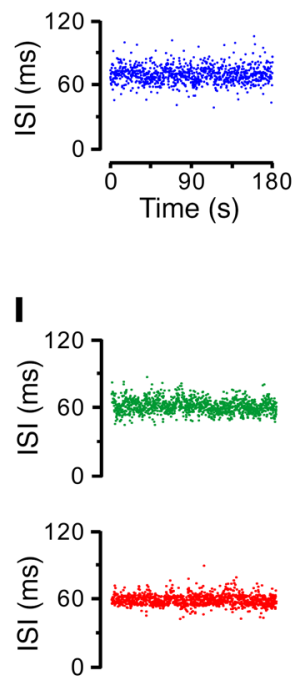

C
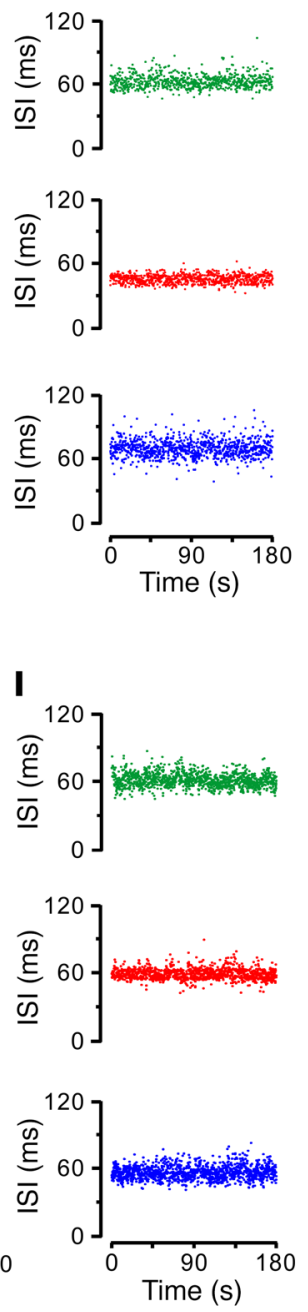
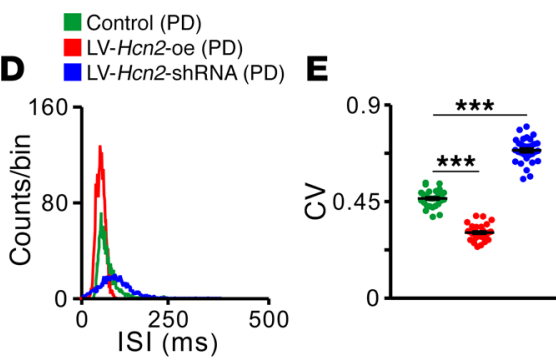

F
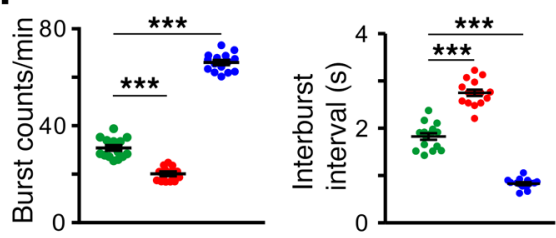

Figure 6. Overexpression of the HCN2 channel, as well as STN-DBS, regularizes firing patterns of STN neurons in PD rats. (A) Three continuous oscilloscope traces show the firings of 3 STN neurons in a PD rat, a PD rat with overexpression of HCN2 in STN, and a PD rat with downregulation of HCN2. Insets represent 5 superimposed traces of spike waveforms for each unit. (B-D) Autocorrelation histograms, scatter plots of ISI series, and ISI histograms of 3 STN neurons presented in A. (E and F) Overexpression of HCN2 in the STN decreased the CV of ISIs and the number of bursts and increased interburst intervals of STN neurons, whereas downregulation of HCN2 increased their CV of ISIs and the number of bursts and decreased interburst intervals (E, $n=30 ; \mathbf{F}, n=15)$. (G) Three continuous oscilloscope traces show the firings of an STN neuron in a PD rat before, during, and after high-frequency stimulation ( $125 \mathrm{~Hz}, 70 \mu \mathrm{A}, 80 \mu$ s pulse width). Red arrowheads in the zoomed-in portion of the spike train during DBS indicate firing spikes of the recorded STN neuron. Insets represent 15 superimposed traces of spike waveforms for the unit, i.e., 5 traces before (green), 5 traces during (red), and 5 traces after (blue) stimulation. (H-J) Autocorrelation histograms, scatter plots of ISI series, and ISI histograms of the STN neuron presented in G. (K and L) DBS decreased the CV of ISIs $(n=25)$ and the number of bursts and increased interburst intervals of STN neurons $(n=15)$, indicating DBS regularizes the firing pattern of STN neurons. Data are represented as mean \pm SEM. ${ }^{* * *} P<0.001,1$-way ANOVA with Newman-Keuls post hoc test (E, F, K and $\left.\mathbf{L}\right)$.

54). In contrast, we find that the $\mathrm{HCN}$ channel coupled to the $\mathrm{H} 2$ receptor substantially mediates the histamine-induced regularization of firing patterns of STN neurons and improvement of motor deficits in PD rats (Figures 3-5), strongly suggesting a key position of the HCN channel in STN neuronal firing patterns. Although our data and previous study (53) show that 4 subtypes of HCN channels (HCN1-4) have all been expressed in the STN, only the HCN2 channel contributes to the amelioration of PD motor deficits by histamine/histaminergic afferent inputs. Furthermore, it is important to indicate that upregulation of HCN2 increases the regularity of STN neuronal firing patterns in PD rats, while downregulation makes the irregular firing patterns much worse (Figure 6, A-F, and Figure 7, C-J) and consequently abolishes both the histamineinduced (Figure 5, F-H) and the DBS-evoked (Figure 7A) amelioration of parkinsonian motor dysfunction. Given the facts that HCN2 promotes single-spike activity rather than burst firing $(19,55)$ and that suppression of burst-generating capacity in STN remedies bradykinesia $(21,36)$, we suggest that the HCN2 channel may hold an essential position in normal rhythm patterns of STN neuronal firing activity and subsequent motor function of basal ganglia.

Interpretations of therapeutic mechanisms underlying the DBS in STN for treatment of motor dysfunctions in PD remain 
A Vector-shRNA Vector-shRNA LV-Hcn2-shRNA

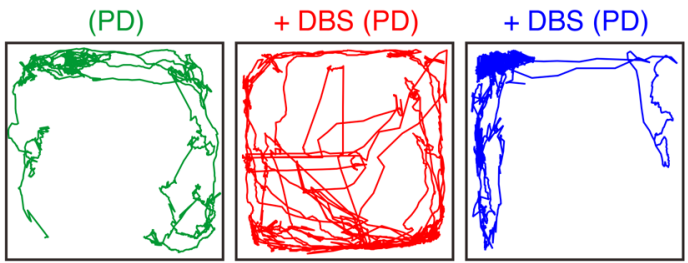

C

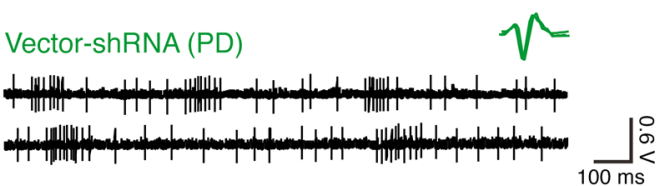

Vector-shRNA + DBS (PD)
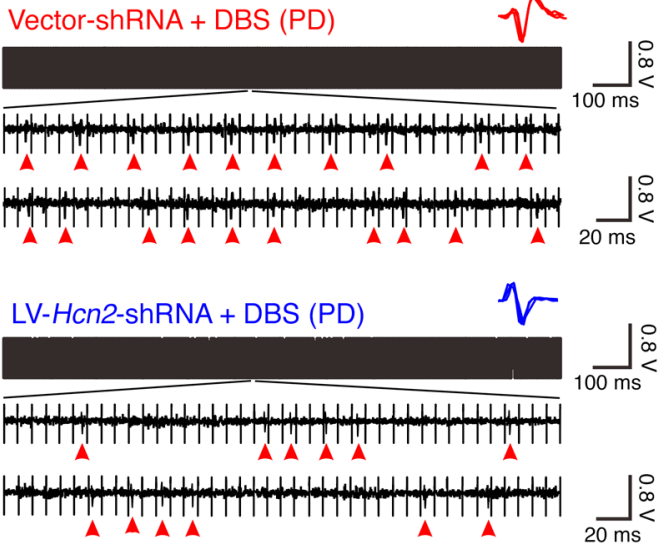
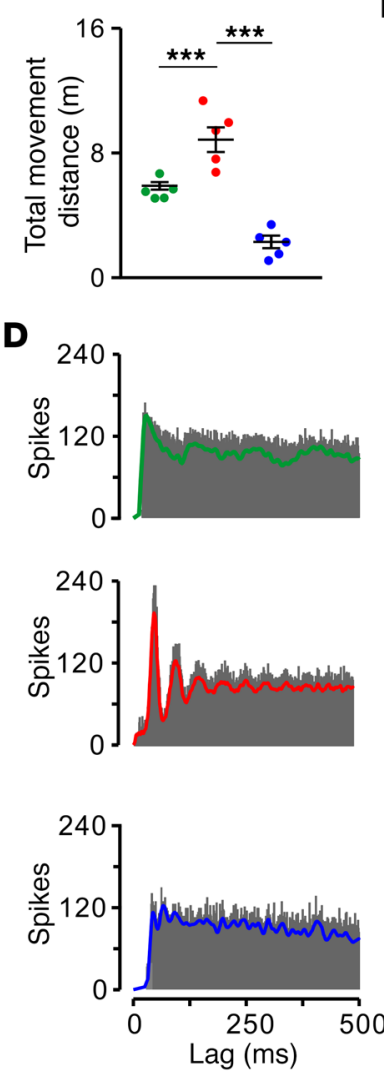

B

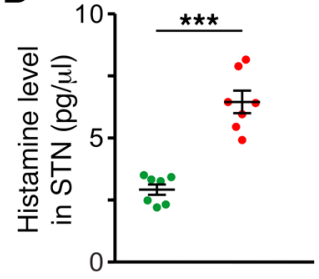

E
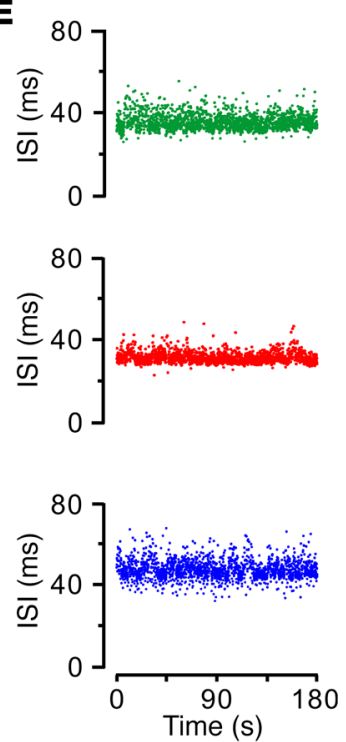

F
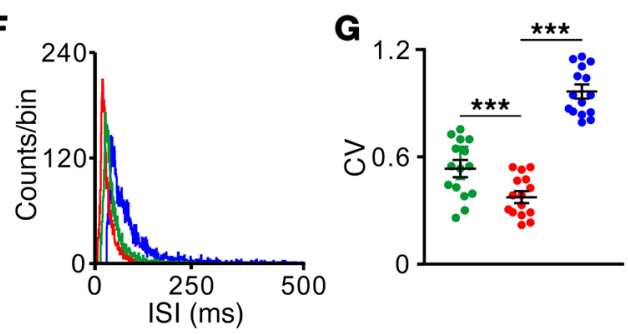

I

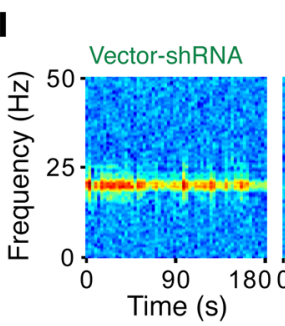

Vector-shRNA + DBS

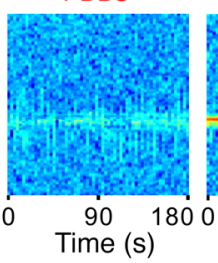

LV-Hcn2-shRNA + DBS

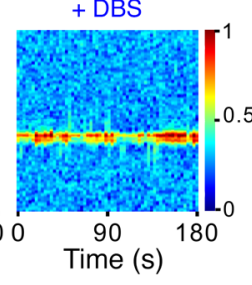

H

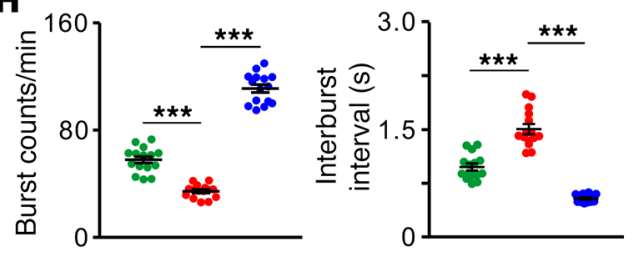

Figure 7. Downregulation of HCN2 attenuates STN-DBS-induced amelioration of motor dysfunction, firing patterns, and $\beta$ oscillations in free-moving PD rats. (A) Locomotor traces of PD rats in an open field. STN-DBS increased total movement distance of PD rats, whereas downregulation of HCN2 in STN abolished DBS-induced motor amelioration $(n=5)$. (B) STN-DBS increased histamine release in STN of PD rats in open field ( $n=7)$. (C) Three continuous oscilloscope traces show firings of 3 STN neurons in free-moving PD rats treated with control virus, control virus plus STN-DBS, or downregulation of HCN2 virus plus STN-DBS. Red arrowheads indicate firing spikes of recorded STN neurons during DBS. Insets represent 5 superimposed traces of spike waveforms for each unit. (D-F) Autocorrelation histograms, scatter plots of ISI series, and ISI histograms of 3 STN neurons presented in C. (G and H) STN-DBS decreased CV of ISIs and number of bursts and increased interburst intervals of STN neurons in free-moving PD rats, whereas downregulation of HCN2 blocked STN-DBS-induced regularization of neuronal firing patterns $(n=15)$. (I) Power spectrograms of simultaneously recorded local field potentials in STN of these free-moving PD rats in the open field. (J) Power spectral distribution of local field potentials recorded from STN. Gray box indicates the classic $\beta$ band (15-25 Hz). A clear increase of power in the $\beta$ band was found in the PD rats. STN-DBS significantly alleviated dominant $\beta$ band oscillatory activities in PD rats, whereas downregulation of HCN2 abolished STN-DBS-induced suppression of excessive $\beta$ oscillations ( $n=15$ ). Data are represented as mean \pm SEM. ${ }^{* *} P<0.001,1$-way ANOVA with Newman-Keuls post hoc test $(\mathbf{A}, \mathbf{G}, \mathbf{H}$, and $\mathbf{J})$ and 2-tailed paired $t$ test (B). 
controversial, with the prevalent view shifting from the idea that such mechanisms work by inhibition of excessive STN activity (3, 17) to the belief that they do so by activation of cortical afferents/ passing fibers to the $\operatorname{STN}(10,11)$. But electrical stimulation of the cortex for alleviation of PD motor symptoms in humans has proven disappointing (2). Although many studies have suggested a close correlation between the abnormal $\beta$ network oscillations and PD pathophysiology as well as STN-DBS (13-16, 18, 20, 44-46, $56)$, there is little direct evidence to establish the exact role of STN neuronal firing patterns in the DBS therapeutic effect. Our data, in implicating DBS as directly increasing endogenous histamine release and subsequently regularizing STN neuronal firing patterns and suppressing excessive $\beta$ network oscillations to ameliorate parkinsonian motor impairments via the HCN2 channel (Figure 6, G-L and Figure 7), provide an insight into understanding the molecular and neuronal mechanism of STN-DBS. Even though the actual DBS mechanisms may be extraordinarily complex and involve contributions of various afferents to the STN, the fact that regularization of firing patterns of STN neurons substantially alleviates parkinsonian deficits in movement points out the importance of relative regularity of STN neuronal firing patterns in the realization of normal motor function of basal ganglia.

In conclusion, our results demonstrate that histamine and the central histaminergic system are indispensable for maintaining normal STN neuronal firing patterns and basal ganglia motor functions. Our findings also provide clear evidence that DBS ameliorates parkinsonian motor symptoms by directly modulating firing patterns of STN neurons. In the light of the key position of STN neuronal firing patterns, targeting neuronal firing patterns of STN for developing new drugs as well as DBS parameter settings may help to optimize strategies for the treatment of PD motor dysfunctions.

\section{Methods}

Animal model of parkinsonism and pharmacological manipulation. Adult male Sprague-Dawley rats ( 8 weeks of age, weighing 250-300 g; Experimental Animal Center of Nanjing Medical University, Nanjing, China) were used and individually housed under controlled environmental conditions $\left(22 \pm 2^{\circ} \mathrm{C} ; 60 \% \pm 5 \%\right.$ humidity; and 12-hour light/12-hour dark cycle with lights on at 8:00 am daily). The animals had free access to standard laboratory chow and water. All rats were naive at the initiation of experiments.

We created unilateral 6-OHDA (MilliporeSigma) lesions to establish a rat model of hemiparkinsonism. After anesthesia induced by intraperitoneal injection of sodium pentobarbital $(40 \mathrm{mg} / \mathrm{kg})$, adult rats weighing 250-300 g were placed into a stereotaxic frame (1404, David Kopf Instruments) for the 6-OHDA injection in the medial forebrain bundle (A, $-4.4 \mathrm{~mm} ; \mathrm{L}, 1.8 \mathrm{~mm}$; and $\mathrm{H}, 7.8 \mathrm{~mm}$ ), according to the rat brain atlas of Paxinos and Watson (57), under aseptic conditions. Rats were pretreated with desipramine hydrochloride $(25 \mathrm{mg} / \mathrm{kg}$, i.p.; MilliporeSigma) 30 minutes before 6-OHDA injection to protect noradrenergic neurons. A stainless steel cannula (outside diameter [o.d.], $0.5 \mathrm{~mm}$; inside diameter [i.d.], $0.3 \mathrm{~mm}$ ) was connected by a polyethylene catheter to a $10 \mu \mathrm{l}$ Hamilton syringe driven by an infusion pump (KDS100, KD Scientific). A total volume of $4 \mu \mathrm{l}$ of the 6-OHDA solution $(2.5 \mu \mathrm{g} / \mu \mathrm{l}$, dissolved in saline containing $0.02 \%$ ascorbic acid, i.p.; MilliporeSigma) was then infused over a period of 4 minutes. The cannula was left in place for 10 minutes before withdrawal. Two weeks after surgery, the rats were tested for rotational behavior under injection of apomorphine $(0.25 \mathrm{mg} / \mathrm{kg})$. Only rats with prominent turning behavior (more than 25 turns in 5 minutes; see below for details) contralateral to the lesion side were considered as the parkinsonian rats, which were retained for further electrophysiological and behavioral studies. At the end of the studies, tyrosine hydroxylase (TH) immunostaining in the bilateral substantia nigras was conducted to identify ipsilesional dopaminergic depletion (Supplemental Figure 1).

For pharmacological manipulation, a stainless-steel guide tube (length, $11 \mathrm{~mm}$; o.d., $0.8 \mathrm{~mm}$; i.d., $0.5 \mathrm{~mm}$ ) for the microinjection cannulae was implanted into the STN ipsilateral to the lesion side of parkinsonian rats or bilaterally in normal rats. The lower end of the guide tube was positioned $1.8 \mathrm{~mm}$ above the STN (A, $-3.8 \mathrm{~mm} ; \mathrm{L}, 2.5 \mathrm{~mm}$; and $\mathrm{H}, 8.0 \mathrm{~mm}$ ), according to the rat brain atlas (57). After implantation, rats were kept on a heating pad until recovery and caged individually. During the behavioral testing sessions, 1 or 2 stainless-steel injection cannulae (length, $13 \mathrm{~mm}$; o.d., $0.5 \mathrm{~mm}$; i.d., $0.3 \mathrm{~mm}$ ) were inserted to protrude $2 \mathrm{~mm}$ beyond the tip of the guide tube. Histamine $(1 \mu \mathrm{g}$; MilliporeSigma), high $\mathrm{K}^{+}(0.75 \mu \mathrm{g} \mathrm{KCl})$, 2-PyEA (a selective agonist for the $\mathrm{H} 1$ receptor; $1 \mu \mathrm{g}$; MilliporeSigma), dimaprit (a selective agonist for the $\mathrm{H} 2$ receptor; $2 \mu \mathrm{g}$; Tocris), R-(-)- $\alpha$-methylhistamine (a selective agonist for the $\mathrm{H} 3$ receptor; $1.5 \mu \mathrm{g}$; Tocris), VUF8430 (a selective agonist for the $\mathrm{H} 4$ receptor; $3 \mu \mathrm{g}$; Tocris), mepyramine (a selective antagonist for the $\mathrm{H} 1$ receptor; $4 \mu \mathrm{g}$; Tocris), ranitidine (a selective antagonist for the $\mathrm{H} 2$ receptor; $3.5 \mu$ g; Tocris), JNJ5207852 (a selective antagonist for the H3 receptor; $2 \mu$ g; Tocris), JNJ7777120 (a selective agonist for the H4 receptor; $2.5 \mu \mathrm{g}$; Tocris), ZD7288 (a selective blocker for the HCN channel; $3 \mu \mathrm{g}$; Tocris), or normal saline $(0.9 \% \mathrm{NaCl})$ was unilaterally or bilaterally microinjected with a Hamilton syringe or syringes $(0.5$ $\mu$ each side, lasting 2 minutes). The effective extent of the drug diffusion in the present study was restricted in the STN according to the estimate by extracellular electrophysiological recording of single-unit activity of neurons located $0.05-0.6 \mathrm{~mm}$ away from the injection sites, as we previously reported (58). Data from rats in which the injection sites were histologically identified $(58,59)$ to be deviated from the STN were excluded from further analysis.

Anterograde tracing with BDA. As previously described (59-61), a glass micropipette (WPI) with an inner tip diameter of 10-15 $\mu \mathrm{m}$ was filled with $10 \%$ biotinylated dextran amine (BDA) (D-1956, Life Technologies) in 0.01 M PBS (pH 7.4) and lowered slowly into the TMN of the hypothalamus at the coordinates $\mathrm{A},-4.0 \mathrm{~mm}$; $\mathrm{L}, 1.6 \mathrm{~mm}$; and $\mathrm{H}$, $9.2 \mathrm{~mm}$, according to the rat brain atlas (57). Iontophoresis as used under $5 \mu$ A positive alternating current ( 7 seconds on $/ 7$ seconds off) for 40 minutes. Once completed, the micropipette was left in place for 10 minutes before removal. During micropipette withdrawal, the current was reversed to minimize tracer leakage through the injection tract. Rats were allowed to survive for 3 weeks before the terminal experiment was conducted.

Lentivirus production and injection. To downregulate the expression of individual HCN channel subtypes in the STN, 4 shRNA lentiviruses for $\mathrm{Hcn}$ (Hcn1 to Hcn4) targeting rat HCN mRNA were constructed (Genechem). The sequences used against rat $\mathrm{Hcn}$ subtypes were as follows: Hcn1, GCCCGGAGACTATATCATT; Hcn2, CCAAGATCCTCAGTCTGCT; Hcn3, AGACATGGCTCGTGGTATT; and Hcn4, GGAAGAGGATGGTGAGGAA. Recombinant lentiviruses LV-Hcn1-shRNAEGFP, LV-Hcn2-shRNA-EGFP, LV-Hcn3-shRNA-EGFP, and LV-Hcn4shRNA-EGFP were produced by cotransfecting $293 \mathrm{~T}$ cells with the 
lentivirus expression plasmid and packaging plasmids using Lipofectamine 2000. To upregulate the expression of $\mathrm{Hcn} 2$, the $\mathrm{Hcn} 2$ expression vector was constructed by inserting their ORF sequence into the pGCL-EGFP vector. The vector containing a control shRNA for downregulation of HCN subtypes and the vector lacking an insert for overexpression of HCN2 channel were used as controls. All correct insertions were confirmed by restriction mapping and direct DNA sequencing. Transfection was performed according to the manufacturer's protocol. Infectious lentiviruses were harvested 72 hours after transfection, and viral titers were $1 \times 10^{9} \mathrm{TU} / \mathrm{ml}$.

The concentrated lentivirus was delivered to the ipsilesional STN of the 6-OHDA-lesioned rat model of parkinsonism or bilaterally in normal rats using a $1 \mu \mathrm{l}$ Hamilton syringe with a thin 25-gauge metal needle, and the injection was driven by an infusion pump (KDS100, KD Scientific; injection volume and flow rate: $1 \mu \mathrm{l}$ at $0.1 \mu \mathrm{l} / \mathrm{min})$. After the injection, the needle was left in place for 10 additional minutes and then slowly withdrawn. For parkinsonian rats, the above procedure was conducted on 7 days after 6-OHDA injection. The rats treated with lentivirus were caged individually and allowed to recover for 7 days before further electrophysiological and behavioral studies. The downregulation or upregulation of expression of HCN channel subtypes in the STN was assessed by qPCR and Western blot.

Analyses of histamine and dopamine level. The level of histamine in the STN was determined by HPLC. In brief, the tissue was homogenized in $1 \mathrm{mM}$ Tris, $1 \mathrm{mM}$ EGTA, $1 \mathrm{mM}$ DTT, and 10\% sucrose, pH 7.5. After centrifugation, the supernatant was removed and a total of $10 \mathrm{ml}$ of the filtrate was injected onto the analytical column. Analyses were performed on an Agilent Series 1100 liquid chromatograph (Agilent Technologies) equipped with a vacuum degasser, a quaternary pump, an autosampler, and a diode-array detector, connected to Agilent ChemStation software (Agilent Technologies).

In addition, the histamine level in the STN and dopamine level in the substantia nigra were quantified by competitive ELISA. In brief, the tissue was homogenized in cool PBS $\left(4^{\circ} \mathrm{C}, \mathrm{pH} 7.2\right)$. After centrifugation, the supernatant was filter sterilized with $0.22 \mu \mathrm{m}$ pore-size polyvinylidene difluoride filters (MilliporeSigma). Histamine and dopamine concentrations were determined using histamine (Cayman Chemical) and dopamine (Biovision) ELISA kits according to the manufacturers' instructions. Absorbance was read at $410 \mathrm{~nm}$ on a Biotek ELx50 spectrophotometer (Biotek), and concentration was calculated using an equation generated from a standard curve.

Immunohistochemistry and stereology. The experimental procedures for immunohistochemistry and stereology followed our previous reports (59-61). Briefly, rats were deeply anesthetized with sodium pentobarbital $(65 \mathrm{mg} / \mathrm{kg})$ and perfused transcardially with $100 \mathrm{ml}$ normal saline, followed by $250-300 \mathrm{ml} 4 \%$ paraformaldehyde or $4 \% N$-(3-dimethylaminopropyl)- $N$-ehylcarbodiimide hydrochloride (EDAC) (MilliporeSigma; for histamine immunostaining experiments) in $0.1 \mathrm{M}$ phosphate buffer. Subsequently, the brain was removed, trimmed, and postfixed in the same fixative for 12 hours at $4^{\circ} \mathrm{C}$ and then cryoprotected with $30 \%$ sucrose for 48 hours. For tissue processed for histamine and/or glutamate immunoreactivity, the brain was postfixed in $4 \%$ EDAC for 4 hours, followed by $4 \%$ glutaraldehyde in $0.1 \mathrm{M}$ phosphate buffer. Frozen coronal sections ( $25 \mu \mathrm{m}$ thick) containing the STN, TMN, or substantia nigra were obtained by a freezing microtome (CM3050S, Leica) and mounted on gelatin-coated slides. The slices were rinsed with $0.1 \mathrm{M}$ PBS, pH 7.2, containing 0.1\% Triton
$\mathrm{X}-100$ and then incubated in $10 \%$ normal bovine serum in PBS containing 0.1\% Triton X-100 for 30 minutes. For glutamate immunoreactivity, slices were treated with $0.5 \%$ sodium borohydride in PBS for 30 minutes to quench autofluorescence produced by glutaraldehyde, followed by repeated washes.

Sections were incubated overnight at $4^{\circ} \mathrm{C}$ with primary antibody/ antibodies, as follows: mouse anti-glutamate (1:1000, MilliporeSigma; catalog MAB5304, RRID:AB_94698), goat anti-GAD67 (1:1000, Abcam; catalog ab80589, RRID:AB_1640532), rabbit anti-histamine (1:1000, MilliporeSigma; catalog AB5885, RRID:AB_177540) and rabbit anti-histamine (1:500, Acris; catalog 22939, RRID:AB_572245), mouse anti-TH (1:2000, MilliporeSigma; catalog T2928, RRID:AB_477569), goat anti-H1 receptor (1:500, Everest Biotech; catalog EB06904, RRID:AB_2230568) and rabbit anti-H1 receptor (1:50, Santa Cruz Biotechnology Inc.; catalog SC20633, RRID:AB_2277328), goat anti-H2 receptor (1:500, Everest Biotech; catalog EB06905, RRID:AB_2121375), rabbit anti-H4 receptor (1:200, Santa Cruz Biotechnology Inc.; catalog SC50313, RRID:AB_2119026), rabbit anti-HCN1 (1:300, Alomone Labs; catalog APC-056, RRID:AB_2039900), rabbit anti-HCN2 (1:100, Alomone Labs; catalog APC-030, RRID:AB_2313726), rabbit antiHCN3 (1:100, Alomone Labs; catalog APC-057, RRID:AB_2039904), and rabbit anti-HCN4 (1:200, Alomone Labs; catalog APC-052, RRID:AB_2039906). These primary antibodies were validated for species and application (1DegreeBio and Antibody Registry). After a complete wash in PBS, the sections for single, double, and triple immunostaining were incubated in the related secondary antibodies (1:2000; Life Technologies) conjugated to Alexa Fluor 488, Alexa Fluor 594, and/or Alexa Fluor 350 for 2 hours at room temperature in the dark. The slides were washed and mounted in Fluoromount-G mounting medium (SouthernBiotech). Incubations replacing the primary antiserum with control immunoglobulins and/or omitting the primary antiserum were used as negative controls. All micrographs were taken with an inverted laser-scanning confocal FluoView FV1000 microscope (Olympus) equipped with Plan-Apochromat $\times 60 / 1.42$ NA oil, $\times 40 / 0.9$ NA dry, $\times 20 / 0.75$ NA dry, and $\times 10 / 0.4$ NA dry objective lenses. Digital images from the microscope were recorded with FV10-ASW 3.1 Viewer Software (Olympus), and image processing was done with Photoshop (Adobe Inc.) and Image Pro Plus (6.0) software (Media Cybernetics).

The number of the dopaminergic neurons in the substantia nigra pars compacta and the EGFP-positive glutamatergic neurons (in which HCN channels were selectively downregulated or upregulated by lentivirus vectors) in the STN were quantified by numerical density, which was estimated by counting the number of neurons within 3D optical dissectors that were systematically spaced at random throughout the selected brain areas. Ten optical dissectors sized $100 \times 100$ $\times 50 \mu \mathrm{m}$ were randomly sampled, and the number of positive cells in each dissector was quantified. The density of cells was estimated using the following formula: $N v=Q / v$ (dis), where $Q$ is the average number of cells counted per dissector, and $v$ (dis) is the volume of the dissector: $v($ dis $)=a$ [frame $] \times h$, where $a$ is area of frame and $h$ is dissector height. Data were represented as the number of cells per cubic millimeter. The histaminergic fiber density in STN was measured by calculation of areas covered with fibers in 20 optical dissectors sized $100 \times 100$ $\mu \mathrm{m}$ that were systematically spaced at random throughout the STN.

qPCR on tissue punches and single-cell qPCR. qPCR on tissue punches and single-cell qPCR were performed as previously described $(30,58)$. For quantification, the quantity of the target gene was expressed rela- 
tive to the amount of the reference gene (Gapdh) to obtain a normalized target expression value. For negative controls, cDNA was replaced with water. Primer sequences are summarized in Supplemental Table 1.

For single-cell qPCR, after whole-cell patch clamp recordings, the content of the recorded neuron was aspirated into the recording pipette and expelled into Single Cell Lysis/Dnase I solution using the Single Cell-to-CT Kit (Life Technologies). Reverse transcription and cDNA preamplification were performed on ABI QuantStudio 6 Flex (Life Technologies) according to the kit protocol. qPCR was performed using the TaqMan Gene Expression Assay system. The TaqMan assay probes were designed by and purchased from Life Technologies as follows: Rn00566691_s1 for Hrh1, Rn00564216_s1 for Hrh2, Rn00585276_m1 for Hrh3, Rn00590929_m1 for Hrh4, Rn00670384_ $\mathrm{m} 1$ for Hcn1, Rn01408572_mH for Hcn2, Rn00586666_m1 for Hcn3, Rn00572232_m1 for Hcn4, and Rn01775763_g1 for Gapdh. Conditions for the cycles followed the manufacturer's protocol for TaqMan assays. A negative control was obtained from clean pipettes containing internal solution. Results were analyzed by using Real-Time StatMiner software (Integromics).

Western blot. STN tissue punches were homogenized in $200 \mu \mathrm{l}$ lysis buffer (1\% Nonidet P-40, $20 \mathrm{mM}$ Tris, pH 8.0, $137 \mathrm{mM} \mathrm{NaCl}$, $10 \%$ glycerol, $1 \mathrm{mM}$ PMSF, sodium butyrate $1 \mathrm{mM}$, and protease inhibitors) at $4^{\circ} \mathrm{C}$. After removal of cellular debris by centrifugation, the supernatant was collected, and protein levels were measured by the Bradford assay (Bio-Rad). For each sample, $20 \mu \mathrm{g}$ was boiled in the presence of sample buffer for 5 minutes before separation on $10 \%$ SDS polyacrylamide gel, and proteins were transferred to nitrocellulose membranes. The immunoblots were blocked with $5 \%$ milk in TBS for 60 minutes. The membranes were then incubated overnight at $4^{\circ} \mathrm{C}$ with primary antibodies as follows: rabbit anti-HCN1 (1:500, Alomone Labs), rabbit anti-HCN2 (1:200, Alomone Labs), rabbit anti-HCN3 (1:200, Alomone Labs), or rabbit anti-HCN4 (1:300, Alomone Labs) (clone number provided earlier). Primary antibody incubation was followed by 3 washes ( 5 minutes, rocking, room temperature) in TBST (TBS containing $0.2 \%$ Tween 20 ) before incubation with the secondary antibody (HRP-conjugated goat anti-rabbit IgG $[\mathrm{H}+\mathrm{L}]$ secondary antibody; Thermo Fisher Scientific), followed by 3 washes. The protein-antibody complexes were visualized by the Pierce ECL Western Blotting Substrate (Thermo Fisher Scientific) and exposed to Kodak medical x-ray film (Denville Scientific Inc.). GAPDH was used as a loading control. The optical densities of protein bands were quantitatively analyzed with Quantity One software (Bio-Rad).

Multielectrode array recordings in vivo. To record the extracellular neuronal activities of STN in vivo, elliptic microwire recording arrays $\left(\sim 0.628 \mathrm{~mm}^{2}\right.$; Stablohm 675, Formvar natural coated, California A Fine Wire), constructed of 14 microwires for recording and a pair of bipolar/twisted electrodes (stainless steel, Teflon coated, $100 \mu \mathrm{m}$ in diameter) in the center for stimulation, were used and targeted at ipsilesional STN (A, -3.4 to $-3.9 \mathrm{~mm}$; L, 2.2 to $2.8 \mathrm{~mm}$; and $\mathrm{H}, 7.3$ to $8.3 \mathrm{~mm}$ ) in parkinsonian rats, according to the rat brain atlas (57). An additional ground wire, which was firmly attached to the skull, was connected to the stainless steel screw for reference.

Single-unit neuronal activity was recorded using a data acquisition system (Cerebus, Blackrock Microsystems). The STN neuronal discharges were detected by the electrode array and passed from the headstage assemblies to the amplifier. The STN was identified by its highly typical firing properties distinguished from cells of the overly- ing zona incerta and the underlying cerebral peduncle. Penetration of the electrode tip into the STN is characterized by a sudden increase in single-unit activity of spontaneously active neurons, and the exit of electrode tip out of the STN corresponds to a loss of single-cell activity $(10,62)$. The analog signals were amplified and filtered at cut-off frequencies of $0.3 \mathrm{~Hz}$ and $7.5 \mathrm{kHz}$. The signals from each microelectrode were further filtered $(250 \mathrm{~Hz}$ to $5 \mathrm{kHz}$ ) and continuously digitized and saved to disk at a rate of $30 \mathrm{kHz}$. Then the recorded data were analyzed by Spike 2 (CED) and NeuroExplorer (MicroBrightField). Only single units with clear separation from the noise cluster were used for further analysis. Spike waveforms were sorted, using a combination of automatic and manual sorting techniques. Automatic clustering techniques (K-means clustering and valley seeking methods) were used to produce an initial separation of waveforms into individual clusters. Each cluster was then checked manually to ensure that the cluster boundaries were well separated and spike waveforms were consistent. A group of similar waveforms was considered as being generated from a single neuron. The peristimulus time histograms (PSTHs) (bin width $1 \mathrm{~s}$ ) of neuronal discharges were generated to assess the changes in firing rate of STN neurons. The autocorrelograms of unit activity (bin width $1 \mathrm{~ms}$ ) was smoothed with Gaussian filter (the solid curve is Gaussian fit to the data), ISI time series (scatterplot), and ISI distributions (bin width $1 \mathrm{~ms}$ ) were generated to analyze firing patterns. Firing bursts were defined by the following parameters: maximal interval at start of a burst, $200 \mathrm{~ms}$; maximal ISI in a burst, $400 \mathrm{~ms}$; minimal duration of a burst, $100 \mathrm{~ms}$; minimal interval between bursts, $200 \mathrm{~ms}$; minimal number of spikes in a burst, 3. The CV of ISI, the number of bursts, and the interburst intervals during the period between 90 seconds before and 90 seconds after maximal firing rate were counted and calculated for assessing the degree of regularization of firing patterns.

The local field potentials were simultaneously recorded through recording electrodes. STN oscillatory rhythm was analyzed using NeuroExplorer software. Power spectral density was built to observe the power distribution under different conditions. The spectrum value was normalized as log of raw power spectral density from 0.5 to 100 $\mathrm{Hz}$, which was calculated using fast Fourier transform with Hanning window function, shifting each 0.05 seconds without overlap. The frequency block was set at 512 at $0.2 \mathrm{~Hz}$ resolution.

Patch-clamp recordings in vitro. Whole-cell patch-clamp and cell-attached recordings were performed as previously described (58, 59,63 ) on STN neurons on brain slices to assess the receptor and ionic mechanisms. Cell-attached recordings were carried out to analyze firing rates and firing patterns without rupturing the cell membrane. The artificial cerebrospinal fluid (ACSF) used for whole-cell patchclamp recordings was as follows: $124 \mathrm{mM} \mathrm{NaCl}, 2.5 \mathrm{mM} \mathrm{KCl}, 1.25 \mathrm{mM}$ $\mathrm{NaH}_{2} \mathrm{PO}_{4}, 1.3 \mathrm{mM} \mathrm{MgSO}_{4}, 26 \mathrm{mM} \mathrm{NaHCO}_{3}, 2 \mathrm{mM} \mathrm{CaCl}_{2}$, and $20 \mathrm{mM}$ D-glucose. That for cell-attached recordings was as follows: $124 \mathrm{mM}$ $\mathrm{NaCl}, 5 \mathrm{mM} \mathrm{KCl}, 1.25 \mathrm{mM} \mathrm{KH}_{2} \mathrm{PO}_{4}, 1.3 \mathrm{mM} \mathrm{MgSO}_{4}, 26 \mathrm{mM} \mathrm{NaHCO}_{3}$, $2.4 \mathrm{mM} \mathrm{CaCl}$, and $10 \mathrm{mM} \mathrm{D}$-glucose. That for high potassium in whole-cell patch-clamp recordings was as follows (substituting part of the $\mathrm{KCl}$ with equimolar $\mathrm{NaCl}$ ): $120.25 \mathrm{mM} \mathrm{NaCl}, 8.75 \mathrm{mM} \mathrm{KCl}, 1.25$ $\mathrm{mM} \mathrm{KH}_{2} \mathrm{PO}_{4}, 1.3 \mathrm{mM} \mathrm{MgSO}_{4}, 26 \mathrm{mM} \mathrm{NaHCO}_{3}, 2.4 \mathrm{mM} \mathrm{CaCl}_{2}$, and 10 $\mathrm{mM}$ D-glucose.

STN neurons were visualized with an Olympus BX51WI microscope equipped with infrared differential interference contrast. Wholecell patch-clamp and cell-attached recordings were acquired with an Axopatch-700B amplifier (Axon Instruments), and the signals were 
fed into the computer through a Digidata-1550 interface (Axon Instruments) for data capture and analysis (pClamp 10.0, Axon Instruments). We bathed the slices with histamine $(0.3-30 \mu \mathrm{M})$ and high $\mathrm{K}^{+}$to stimulate the recorded STN neurons. Before bath application, the whole-cell current or spontaneous firing rate of the recorded neuron was observed for at least 20 minutes to assure stability. Then histamine or high $\mathrm{K}^{+}$ was added to the perfusing ACSF to stimulate the recorded neuron for a test period of 1 minute. After each stimulation, cells were given at least 20 minutes for recovery and prevention of desensitization. TTX (0.3 $\mu \mathrm{M}$, Alomone Labs), selective non-NMDA receptor antagonist NBQX (20 $\mu \mathrm{M}$, Tocris), selective NMDA receptor antagonist AP5 $(50 \mu \mathrm{M}$, Tocris), and selective $\mathrm{GABA}_{\mathrm{A}}$ receptor antagonist SR 95531 (50 $\mu \mathrm{M}$, Tocris) were used to determine whether the effect of histamine was postsynaptic. Selective agonists for histamine receptor subtypes 2-PyEA $(30 \mu \mathrm{M})$, dimaprit $(30 \mu \mathrm{M})$, and VUF8430 $(30 \mu \mathrm{M})$ as well as selective antagonist mepyramine $(1 \mu \mathrm{M})$, ranitidine $(1 \mu \mathrm{M})$, or JNJ7777120 $(10 \mu \mathrm{M})$ was applied to examine the underlying postsynaptic receptor mechanism. The receptor antagonist or ion channel blocker was given for at least 15 minutes before we observed effects.

To characterize the histamine-induced whole-cell current, in voltage-clamp recording, current-voltage plots ( $I$ - $V$ curves) were obtained before and during histamine application using a slow ramp command $(d V / d t=-10 \mathrm{mV} / \mathrm{s}$, ranged from -60 to $-130 \mathrm{mV})$ to allow for attainment of steady-state conditions $(58,59)$. In addition, depolarizing voltage sag generated by activation of the HCN channels in response to a hyperpolarizing current stimulation ( $80-150 \mathrm{pA}, 1$ second) in the absence and presence of histamine was measured in current-clamp recordings. The amplitude of voltage sag was calculated by subtracting the peak voltage amplitude from the steady-state voltage, and ZD7288 $(50 \mu \mathrm{M})$ was applied to block HCN channels. Moreover, to examine the effect of histamine on the HCN channel current $\left(I_{h}\right), I-V$ curves were obtained before and during histamine application using a series of 1-second hyperpolarizing voltage steps (ranging from -50 to $-120 \mathrm{mV}$ in $10 \mathrm{mV}$ steps) (64). $I_{h}$ was determined by subtracting instantaneous current $\left(I_{\text {Ins }}\right)$ from maximum current at 1 second $\left(I_{\text {Max-Ims }}\right)$ at each hyperpolarizing voltage step using the following equation: $I_{h}=$ $I_{\text {Max-Ims }}-I_{\text {Ins }} . I_{h}$ conductance $\left(G_{h}\right)$ was estimated as the amplitude of $I_{h}$ measured at various potentials $(V)$ divided by the driving force $\left(V-E_{h}\right)$ : $G_{h}=I_{h} /\left(V-E_{h}\right)$, where $E_{h}$ is the reversal potential of $I_{h}$ determined by clamping STN neurons to $-120 \mathrm{mV}$ for 1 second and depolarizing in $10 \mathrm{mV}$ at 1 -second increments to $-50 \mathrm{mV}$. $G$ was normalized to maximum and fitted with the modified Boltzmann function: $G_{\text {norm }}=1 /(1+$ $\left.\exp \left(\left(V-V_{1 / 2}\right) / k\right)\right)$, where $G_{n o r m}$ is the fraction of maximal $G_{h}$ observed at $V, V_{1 / 2}$ is the voltage required for half-maximal activation, and $k$ is the slope factor.

DBS of STN. A pair of bipolar/twisted stimulating electrodes (stainless steel, Teflon coated, $100 \mu \mathrm{m}$ in diameter) in the center of elliptic microwire recording arrays $\left(\sim 0.628 \mathrm{~mm}^{2}\right.$; Stablohm 675 , Formvar natural coated, CA Fine Wire) constructed of 14 microwires for recording was implanted stereotaxically into the ipsilesional STN (A, -3.6 mm; L, $2.5 \mathrm{~mm}$; and H, $8.0 \mathrm{~mm}$ ), with the guidance of electrophysiological recordings for the highly typical firing properties of STN neurons as described in Multielectrode array recordings in vivo. Electrical rectangular stimulation was applied to STN by using a Master-9 Stimulator (AMPI). Stimulation parameters were as follows: frequency, $125 \mathrm{~Hz}$; intensity, $70 \mu \mathrm{A}$; pulse width, $80 \mu \mathrm{s}$; and stimulation time, 5 minutes (11).
In vivo microdialysis sampling. In vivo microdialysis sampling of histamine was performed on freely moving 6-OHDA-lesioned animals. The microdialysis probe had an $8.0 \mathrm{~mm}$ shaft with a $1.0 \mathrm{~mm}$, $50 \mathrm{kD}$ molecular weight cutoff polyethylene membrane (DZ-9-01, Eicom). Before use, the probe was washed with ACSF. The preconditioned probe's inlet was connected to a microsyringe pump (ESP-32, Eicom) using fluorinated ethylene propylene tubing. Probe implantation was performed on anesthetized animals 10 days after lesions were created. Rats were stereotactically implanted with a guide cannula at a 20-degree angle (caudorostral to the vertical plane) into the ipsilesional STN (A, -3.6 mm; L, $2.5 \mathrm{~mm}$; and H, $8.0 \mathrm{~mm}$ ). Four days after surgery, the rats were placed in an open field, and a probe was inserted through the guide. The probe was perfused with ACSF for 240 minutes at a flow rate of $10 \mu \mathrm{l} / \mathrm{min}$ before sample collection. Samples (50 $\mu \mathrm{l})$ were collected at a flow rate of $0.5 \mu \mathrm{l} / \mathrm{min}$ and kept at $4^{\circ} \mathrm{C}$ (EFR-82 Cooling Unit; Eicom). Each hour, a representative sample was transferred to a $-80^{\circ} \mathrm{C}$ freezer and stored until analyzed.

Behavioral tests. The animals used in behavioral tests were randomly grouped by different treatments according to a random number table. All behavioral tests started at the same time (10:00 am) each day. The experimenter performing the behavioral tests was blind to the treatment groups and the side of dopamine depletion. In order to achieve a stable motor performance in the adhesive removal, accelerating rotarod, and balance beam tests, each animal was trained daily for at least 10 trials for 3 to 5 consecutive days before tests, as we previously reported $(58,59)$.

Turning behavior test. The 6-OHDA-lesioned animals were given apomorphine $(0.25 \mathrm{mg} / \mathrm{kg}$, i.p.) and placed individually into a $30 \mathrm{~cm}$ diameter round bowl. The number of rotations contralateral to the lesion side was counted during a 30-minute interval after apomorphine injection.

The adhesive-removal test. This test is considered as an evaluation of motor initiation and execution (65). Prior to surgery, 2 training trials were performed by placing 2 adhesive tapes $(8 \mathrm{~mm} \times 6 \mathrm{~mm})$ on the plantar surface of both forelimbs simultaneously. In the test, the rat may bring one forelimb onto the other forelimb and use its mouth to remove the adhesives. We trained the animals by performing 1 trial per animal per day for 5 days (each trial lasted a maximum of 3 minutes). The trials began 10 days after surgery. At day 15 after 6-OHDA or saline injections, adhesive dots were placed on both forelimbs and the time to remove the tape was recorded. If a rat did not remove either or both stickers within 60 seconds, the animal received a score of 60 seconds.

Footprints. We assessed walking pattern and gait kinematics by a footprint test (59). Rat hind paws were painted with nontoxic inks, and the rats were allowed to spontaneously traverse a clear plexiglass tunnel $(100 \mathrm{~cm} \times 10 \mathrm{~cm} \times 10 \mathrm{~cm})$ ending in a darkened cage. A sheet of white absorbent paper $(100 \mathrm{~cm} \times 10 \mathrm{~cm})$ was placed at the bottom of the track. The resulting tracks provided the spatial relationship of consecutive footfalls from which the stride length and width were measured.

Balance beam test. We evaluated motor balance and coordination by measuring the ability of the animals to traverse a balance beam $190 \mathrm{~cm}$ in length with a diameter of $2.5 \mathrm{~cm}$. A plastic platform $(7 \mathrm{~cm}$ $\times 4 \mathrm{~cm}$ ) was placed at one end of the rod as the start, and a black plastic box $(15 \mathrm{~cm} \times 15 \mathrm{~cm} \times 8 \mathrm{~cm})$ was set at the other end of the rod as a nest for motivating the animal to cross the beam. The apparatus was suspended $90 \mathrm{~cm}$ above a cushion, which protected the fallen animals from injury, and $50 \mathrm{~cm}$ from a wall. Each animal was 
trained daily for at least 10 trials for 3 to 5 consecutive days in order to achieve a stable performance on the balance beam. The time taken to traverse the beam was recorded. The test consisted of 5 consecutive trials. To reduce stress and fatigue, the animals were allowed a 90-second rest between trials.

Accelerating rotarod test. To assess motor coordination and balance, we used a commercially available accelerating rotarod (47750, Ugo Basile). Animals were first habituated to low rotation (4 rpm) for 30 seconds, and then the rod was evenly accelerated up to $40 \mathrm{rpm}$ for 300 seconds (acceleration was $0.12 \mathrm{rpm} / \mathrm{s}$ ). The time taken for the rat to fall from the rotating rod was recorded, with time beyond 300 seconds recorded as 300 seconds. Each animal was trained daily for at least 10 trials for 3 to 5 consecutive days in order to achieve a stable performance on the accelerating rotarod. For the test, each rat was subjected to 3 trials, with a 3-minute resting interval to reduce stress and fatigue.

Open-field test. An open field arena $(50 \mathrm{~cm} \times 50 \mathrm{~cm} \times 50 \mathrm{~cm})$ was used to assess spontaneous motor activity. The spontaneous locomotor activity was monitored by a video camera, and the total movement distance traveled during 5 minutes was quantified by Clever TopScan (Clever Sys Inc.).

Statistics. All data were analyzed with SPSS 17.0 (SPSS). Data were tested for normal distribution and homogeneity of variance. Twotailed Student's $t$ test, 1-way, 2-way, and repeated measures 2-way ANOVA, and Newman-Keuls post hoc testing were employed for statistical analysis. $P$ values of less than 0.05 were considered to be significant. Detailed statistical results for each experiment are summarized in Supplemental Table 2.

Study approval. All experiments were carried out in accordance with the NIH Guide for the Care and Use of Laboratory Animals (National Academies Press, 2011) and were approved by the Experimental Ani- mal Care and Use Committee of Nanjing University. All efforts were made to minimize the number of animals used and their suffering.

\section{Author contributions}

JNZ and JJW conceived and designed the study. QXZ performed tracing, immunostaining, qPCR, Western blot, HPLC, ELISA, and patchclamp recording experiments. GYL performed behavioral experiments. BL performed multielectrode array recording and behavioral experiments. CZZ and KX were involved in behavioral experiments. HZL was involved in electrophysiological experiments. QXZ, GYL, $\mathrm{BL}$, and XYZ analyzed data. JNZ and JJW wrote the paper.

\section{Acknowledgments}

This work was supported by the National Natural Science Foundation of China (NSFC) (grants 31330033, 81671107, 31471112, 91332124, 31771143, 31500848, 31600834, and NSFC/Research Grants Council [RGC] Joint Research Scheme 31461163001); the Ministry of Education of China (Specialized Research Fund for the Doctoral Program of Higher Education [SRFDP]/RGC Earmarked Research Grant [ERG] grant 20130091140003, New Century Excellent Talents [NCET] Program, and Fundamental Research Funds for the Central Universities 020814380071 and 020814380091); and the Natural Science Foundation of Jiangsu Province, China (grants BK2011014, BK20151384, and BK20140599).

Address correspondence to: Jing-Ning Zhu or Jian-Jun Wang, State Key Laboratory of Pharmaceutical Biotechnology and Department of Physiology, School of Life Sciences, Nanjing University, 163 Xianlin Avenue, Nanjing 210023, China. Phone: 86.25.89682714; Email:jnzhu@nju.edu.cn (JNZ); jjwang@nju.edu.cn (JJW).
1. Benabid AL, Chabardes S, Mitrofanis J, Pollak P. Deep brain stimulation of the subthalamic nucleus for the treatment of Parkinson's disease. Lancet Neurol. 2009;8(1):67-81.

2. Eisenstein M. Electrotherapy: shock value. Nature. 2016;538(7626):S10-S12.

3. DeLong MR. Primate models of movement disorders of basal ganglia origin. Trends Neurosci. 1990;13(7):281-285.

4. Parent A, Hazrati LN. Functional anatomy of the basal ganglia. II. The place of subthalamic nucleus and external pallidum in basal ganglia circuitry. Brain Res Brain Res Rev. 1995;20(1):128-154.

5. Calabresi P, Picconi B, Tozzi A, Ghiglieri V, Di Filippo M. Direct and indirect pathways of basal ganglia: a critical reappraisal. Nat Neurosci. 2014;17(8):1022-1030.

6. Nambu A, Tokuno H, Takada M. Functional significance of the cortico-subthalamopallidal 'hyperdirect' pathway. Neurosci Res. 2002;43(2):111-117.

7. Chu HY, Atherton JF, Wokosin D, Surmeier DJ, Bevan MD. Heterosynaptic regulation of external globus pallidus inputs to the subthalamic nucleus by the motor cortex. Neuron. 2015;85(2):364-376.

8. Chu HY, McIver EL, Kovaleski RF, Atherton JF, Bevan MD. Loss of hyperdirect pathway cortico-subthalamic inputs following degeneration of midbrain dopamine neurons. Neuron. 2017;95(6):1306-1318.e5.
9. Plenz D, Kital ST. A basal ganglia pacemaker formed by the subthalamic nucleus and external globus pallidus. Nature. 1999;400(6745):677-682.

10. Gradinaru V, Mogri M, Thompson KR, Henderson JM, Deisseroth K. Optical deconstruction of parkinsonian neural circuitry. Science. 2009;324(5925):354-359.

11. Li Q, et al. Therapeutic deep brain stimulation in Parkinsonian rats directly influences motor cortex. Neuron. 2012;76(5):1030-1041.

12. Sharott A, et al. Activity parameters of subthalamic nucleus neurons selectively predict motor symptom severity in Parkinson's disease. J Neurosci. 2014;34(18):6273-6285.

13. Yang AI, Vanegas N, Lungu C, Zaghloul KA. Beta-coupled high-frequency activity and betalocked neuronal spiking in the subthalamic nucleus of Parkinson's disease. JNeurosci. 2014;34(38):12816-12827.

14. Huebl J, et al. Oscillatory subthalamic nucleus activity is modulated by dopamine during emotional processing in Parkinson's disease. Cortex. 2014;60:69-81.

15. Cagnan H, Duff EP, Brown P. The relative phases of basal ganglia activities dynamically shape effective connectivity in Parkinson's disease. Brain. 2015;138(Pt 6):1667-1678.

16. Wang DD, et al. Subthalamic local field potentials in Parkinson's disease and isolated dystonia: An evaluation of potential biomarkers. Neurobiol Dis.

\section{6;89:213-222.}

17. Bergman H, Wichmann T, DeLong MR. Reversal of experimental parkinsonism by lesions of the subthalamic nucleus. Science. 1990;249(4975):1436-1438.

18. Escobar Sanabria D, et al. Parkinsonism and vigilance: alteration in neural oscillatory activity and phase-amplitude coupling in the basal ganglia and motor cortex. J Neurophysiol. 2017;118(5):2654-2669.

19. Chan CS, et al. HCN channelopathy in external globus pallidus neurons in models of Parkinson's disease. Nat Neurosci. 2011;14(1):85-92.

20. Delaville C, McCoy AJ, Gerber CM, Cruz AV, Walters JR. Subthalamic nucleus activity in the awake hemiparkinsonian rat: relationships with motor and cognitive networks. J Neurosci. 2015;35(17):6918-6930.

21. Pan MK, et al. Neuronal firing patterns outweigh circuitry oscillations in parkinsonian motor control. J Clin Invest. 2016;126(12):4516-4526.

22. Rinne JO, et al. Increased brain histamine levels in Parkinson's disease but not in multiple system atrophy. J Neurochem. 2002;81(5):954-960.

23. Anichtchik OV, Peitsaro N, Rinne JO, Kalimo H, Panula P. Distribution and modulation of histamine $\mathrm{H}(3)$ receptors in basal ganglia and frontal cortex of healthy controls and patients with Parkinson's disease. Neurobiol Dis. 2001;8(4):707-716.

24. Panula P, Nuutinen S. The histaminergic network 
in the brain: basic organization and role in disease. Nat Rev Neurosci. 2013;14(7):472-487.

25. Haas H, Panula P. The role of histamine and the tuberomamillary nucleus in the nervous system. Nat Rev Neurosci. 2003;4(2):121-130.

26. Haas HL, Sergeeva OA, Selbach O. Histamine in the nervous system. Physiol Rev. 2008;88(3):1183-1241.

27. Sutcliffe JG, de Lecea L. Not asleep, not quite awake. Nat Med. 2004;10(7):673-674.

28. Eban-Rothschild A, Giardino WJ, de Lecea L. To sleep or not to sleep: neuronal and ecological insights. Curr Opin Neurobiol. 2017;44:132-138.

29. Zhu JN, Yung WH, Kwok-Chong Chow B, Chan YS, Wang JJ. The cerebellar-hypothalamic circuits: potential pathways underlying cerebellar involvement in somatic-visceral integration. Brain Res Rev. 2006;52(1):93-106.

30. Zhang J, et al. Selective modulation of histaminergic inputs on projection neurons of cerebellum rapidly promotes motor coordination via $\mathrm{HCN}$ channels. Mol Neurobiol. 2016;53(2):1386-1401.

31. Bolam JP, Ellender TJ. Histamine and the striatum. Neuropharmacology. 2016;106:74-84.

32. Shan L, Dauvilliers Y, Siegel JM. Interactions of the histamine and hypocretin systems in CNS disorders. Nat Rev Neurol. 2015;11(7):401-413.

33. Kravitz AV, et al. Regulation of parkinsonian motor behaviours by optogenetic control of basal ganglia circuitry. Nature. 2010;466(7306):622-626.

34. Hutchison WD, et al. Neurophysiological identification of the subthalamic nucleus in surgery for Parkinson's disease. Ann Neurol. 1998;44(4):622-628.

35. Kass JI, Mintz IM. Silent plateau potentials, rhythmic bursts, and pacemaker firing: three patterns of activity that coexist in quadristable subthalamic neurons. Proc Natl Acad Sci U S A. 2006;103(1):183-188.

36. Tai CH, Yang YC, Pan MK, Huang CS, Kuo CC. Modulation of subthalamic T-type $\mathrm{Ca}(2+)$ channels remedies locomotor deficits in a rat model of Parkinson disease. JClin Invest. 2011;121(8):3289-3305.

37. Pape HC. Queer current and pacemaker: the hyperpolarization-activated cation current in neurons. Annu Rev Physiol. 1996;58:299-327.

38. Ludwig A, Zong X, Jeglitsch M, Hofmann F, Biel M. A family of hyperpolarization-activated mammalian cation channels. Nature. 1998;393(6685):587-591.

39. Seifert R, Scholten A, Gauss R, Mincheva A, Lichter P, Kaupp UB. Molecular characterization of a slowly gating human hyperpolarizationactivated channel predominantly expressed in thalamus, heart, and testis. Proc Natl Acad Sci U S A. 1999;96(16):9391-9396.

40. Biel M, Wahl-Schott C, Michalakis S, Zong $\mathrm{X}$. Hyperpolarization-activated cation channels: from genes to function. Physiol Rev. 2009;89(3):847-885.

41. Chen X, Sirois JE, Lei Q, Talley EM, Lynch C, Bayliss DA. HCN subunit-specific and cAMP-modulated effects of anesthetics on neuronal pacemaker currents. J Neurosci. 2005;25(24):5803-5814.

42. Limousin $\mathrm{P}$, et al. Effect of parkinsonian signs and symptoms of bilateral subthalamic nucleus stimulation. Lancet. 1995;345(8942):91-95.

43. Miocinovic S, Somayajula S, Chitnis S, Vitek JL. History, applications, and mechanisms of deep brain stimulation. JAMA Neurol. 2013;70(2):163-171.

44. Hammond C, Bergman H, Brown P. Pathological synchronization in Parkinson's disease: networks, models and treatments. Trends Neurosci. 2007;30(7):357-364.

45. McCarthy MM, Moore-Kochlacs C, Gu X, Boyden ES, Han X, Kopell N. Striatal origin of the pathologic beta oscillations in Parkinson's disease. Proc Natl Acad Sci USA. 2011;108(28):11620-11625.

46. Brocker DT, Swan BD, So RQ, Turner DA, Gross RE, Grill WM. Optimized temporal pattern of brain stimulation designed by computational evolution. Sci Transl Med. 2017;9(371):aah3532.

47. Atherton JF, Menard A, Urbain N, Bevan MD. Short-term depression of external globus pallidussubthalamic nucleus synaptic transmission and implications for patterning subthalamic activity. JNeurosci. 2013;33(17):7130-7144.

48. Li GY, Zhuang QX, Li B, Wang JJ, Zhu JN. [Subthalamic nucleus: from circuits, functions to a deep brain stimulation target for the treatment of Parkinson's disease]. Sheng Li Xue Bao. 2017;69(5):611-622.

49. Levy R, et al. Lidocaine and muscimol microinjections in subthalamic nucleus reverse Parkinsonian symptoms. Brain. 2001;124(Pt 10):2105-2118.

50. Benedetti F, et al. Placebo-responsive Parkinson patients show decreased activity in single neurons of subthalamic nucleus. Nat Neurosci. 2004;7(6):587-588.

51. Lang AE, Obeso JA. Challenges in Parkinson's disease: restoration of the nigrostriatal dopamine system is not enough. Lancet Neurol. 2004;3(5):309-316.
52. Tiligada E, Kyriakidis K, Chazot PL, Passani MB Histamine pharmacology and new CNS drug targets. CNS Neurosci Ther. 2011;17(6):620-628.

53. Atherton JF, et al. Selective participation of somatodendritic HCN channels in inhibitory but not excitatory synaptic integration in neurons of the subthalamic nucleus. J Neurosci. 2010;30(47):16025-16040.

54. Chetrit J, et al. Inhibiting subthalamic D5 receptor constitutive activity alleviates abnormal electrical activity and reverses motor impairment in a rat model of Parkinson's disease. J Neurosci. 2013;33(37):14840-14849.

55. Ludwig A, et al. Absence epilepsy and sinus dysrhythmia in mice lacking the pacemaker channel HCN2. EMBO J. 2003;22(2):216-224.

56. Tan $\mathrm{H}$, et al. Subthalamic nucleus local field potential activity helps encode motor effort rather than force in parkinsonism. J Neurosii. 2015;35(15):5941-5949.

57. Paxinos G, Watson C. The Rat Brain in Stereotaxic Coordinates. San Diego, CA: Academic Press; 2014.

58. Zhang J, et al. A role for orexin in central vestibular motor control. Neuron. 2011;69(4):793-804.

59. Wang Y, et al. Role of corticotropin-releasing factor in cerebellar motor control and ataxia. Curr Biol. 2017;27(17):2661-2669.e5.

60. Li B, Zhuang QX, Gao HR, Wang JJ, Zhu JN. Medial cerebellar nucleus projects to feeding-related neurons in the ventromedial hypothalamic nucleus in rats. Brain Struct Funct. 2017;222(2):957-971.

61. Ji MJ, Zhang XY, Chen Z, Wang JJ, Zhu JN. Orexin prevents depressive-like behavior by promoting stress resilience [published online ahead of print August 7, 2018]. Mol Psychiat. https://doi. org/10.1038/s41380-018-0127-0.

62. Périer C, Tremblay L, Féger J, Hirsch EC. Behavioral consequences of bicuculline injection in the subthalamic nucleus and the zona incerta in rat. JNeurosci. 2002;22(19):8711-8719.

63. Dugladze T, Schmitz D, Whittington MA, Vida I, Gloveli T. Segregation of axonal and somatic activity during fast network oscillations. Science. 2012;336(6087):1458-1461.

64. Friedman AK, et al. Enhancing depression mechanisms in midbrain dopamine neurons achieves homeostatic resilience. Science. 2014;344(6181):313-319.

65 . Bouet $\mathrm{V}$, et al. The adhesive removal test: a sensitive method to assess sensorimotor deficits in mice. Nat Protoc. 2009;4(10):1560-1564. 